\title{
Application of the Restoring Force Surface Method to a Real-life Spacecraft Structure
}

\author{
J.P. Noel, G. Kerschen \\ Space Structures and Systems Laboratory (S3L), Structural Dynamics Research Group \\ Department of Aerospace and Mechanical Engineering, University of Liège, Belgium \\ jp.noel,g.kerschen@ulg.ac.be
}

A. Newerla

European Space Agency (ESTEC), Noordwijk, The Netherlands

alfred.newerla@esa.int

\begin{abstract}
Many nonlinear system identification methods have been introduced in the technical literature during the last thirty years. However, few of these methods were applied to real-life structures. In this context, the objective of the present paper is to demonstrate that the Restoring Force Surface (RFS) method can provide a reliable identification of a nonlinear spacecraft structure. The nonlinear component comprises an inertia wheel mounted on a support, the motion of which is constrained by eight elastomer plots and mechanical stops. Several adaptations to the RFS method are proposed, which include the elimination of kinematic constraints and the regularization of ill-conditioned inverse problems. The proposed methodology is demonstrated using numerical data.
\end{abstract}

\section{INTRODUCTION}

Nonlinear structural dynamics has been studied for a relatively long time, but the first contributions to the identification of nonlinear structural models date back to the 1970s. Since then, numerous methods have been developed because of the highly individualistic nature of nonlinear systems ${ }^{[1]}$. A large number of these methods were targeted to Single-Degree-Of-Freedom (SDOF) systems, but significant progress in the identification of Multi-Degree-Of-Freedom (MDOF) lumped parameter systems has been realized during the last ten or twenty years. To date, simple continuous structures with localized nonlinearities are within reach. Among the well-established methods, there exist

- time-domain methods such as the Restoring Force Surface (RFS) and Nonlinear Auto-Regressive Moving Average with eXogeneous input (NARMAX) methods ${ }^{[2,3]}$;

- frequency-domain methods such as the Conditioned Reverse Path (CRP) ${ }^{[4]}$ and Nonlinear Identification through Feedback of the Output (NIFO) methods ${ }^{[5]}$;

- time-frequency analysis methods such as the Wavelet Transform (WT) ${ }^{[6]}$.

The RFS method, introduced in 1979 by Masri and Caughey ${ }^{[7]}$, constitutes the first attempt to identify nonlinear structures. Since then, many improvements of the RFS method were introduced in the technical literature. Without being comprehensive, 
we mention the replacement of Chebyschev expansions in favour of more intuitive ordinary polynomials ${ }^{[8]}$, the design of optimized excitation signals ${ }^{[9]}$ or the direct use of the state space representation of the restoring force as nonparametric estimate ${ }^{[10]}$.

In theory ${ }^{[11]}$, the RFS method can handle MDOF systems. However, a number of practical considerations diminish this capability and its scope is, in fact, bound to systems with a few degrees of freedom only. For example, Al-Hadid and Wright ${ }^{[12]}$ studied a T-beam structure with well-separated bending and torsion modes. Another extensively studied system of this kind is the automotive damper ${ }^{[13,14]}$.

The objective of the present paper is to demonstrate the usefulness of the RFS method in the particular case of a real-life nonlinear spacecraft structure: the SmallSat spacecraft from EADS-Astrium. Starting from a review of the required ingredients for a RFS-based identification, we will propose solutions to the inherent limits of the method. First, we will simplify the kinematics of the nonlinear device of the spacecraft, termed WEMS, in order to explicitely formulate its dynamic equations. This formulation will be based on the necessary use of the coordinates of its center of gravity. Eventually, we will discuss why our estimation of coefficients is ill-conditioned and how to circumvent this final issue. The whole procedure will then be demonstrated using numerical data.

\section{THE SMALLSAT SPACECRAFT AND ITS FINITE ELEMENT MODELLING}

The SmallSat structure has been conceived as a low cost structure for small low-earth orbit satellite ${ }^{[15]}$. It is a monocoque tube structure which is $1.2 \mathrm{~m}$ long and $1 \mathrm{~m}$ large. It incorporates eight flat faces for equipment mounting purposes, creating an octagon shape, as shown in Figure 1 (a). The octagon is manufactured using carbon fibre reinforced plastic by means of a filament winding process. The structure thickness is $4.0 \mathrm{~mm}$ with an additional $0.25 \mathrm{~mm}$ thick skin of Kevlar applied to both the inside and outside surfaces to provide protection against debris. The interface between the spacecraft and launch vehicle is achieved through four aluminium brackets located around cut-outs at the base of the structure. The total mass including the interface brackets is around $64 \mathrm{~kg}$.

The SmallSat structure supports a telescope dummy composed of two stages of base-plates and struts supporting various concentrated masses; its mass is around $140 \mathrm{~kg}$. The telescope dummy plate is connected to the SmallSat top floor via three shock attenuators, termed SASSA (Shock Attenuation System for Spacecraft and Adaptator) ${ }^{[16]}$, the behaviour of which is considered as linear in the present study. The top floor is a 1 square meter sandwich aluminium panel, with $25 \mathrm{~mm}$ core and $1 \mathrm{~mm}$ skins. Finally, as shown in Figure 1 (c), a support bracket connects to one of the eight walls the so-called Wheel Elastomer Mounting System (WEMS) device which is loaded with an $8 \mathrm{~kg}$ reaction wheel dummy. The purpose of this device is to isolate the spacecraft structure from disturbances coming from reaction wheels through the presence of a soft interface between the fixed and mobile parts. In addition, mechanical stops limit the axial and lateral motion of the WEMS mobile part during launch, which gives rise to nonlinear dynamic phenomena. Figure 1 (d) depicts the WEMS overall geometry, but details are not disclosed for confidentiality reasons.

The Finite Element (FE) model in Figure 1 (b) was created in Samcef software and is used in the present study to conduct numerical experiments. The comparison with experimental measurements revealed the good predictive capability of this model. The WEMS mobile part (the inertia wheel and its cross-shaped support) was modeled as a flexible body, which is connected to the WEMS fixed part (the bracket and, by extension, the spacecraft itself) through four nonlinear connections, labeled NC 1-4 in Figure 1 (d). Black squares signal such connections. Each nonlinear connection possesses

- a nonlinear spring (elastomer in traction plus 2 stops) in the axial direction,

- a nonlinear spring (elastomer in shear plus 1 stop) in the radial direction,

- a linear spring (elastomer in shear) in the third direction.

The spring characteristics (piecewise linear) are listed in Table 1 and are displayed in Figure 1 (e). We stress the presence of two stops at each end of the cross in the axial direction. This explains the corresponding symmetric bilinear stiffness curve. In the 


\begin{tabular}{|c|c|c|c|}
\hline Spring & Clearance & Stiffness of the elastomer plot & Stiffness of the mechanical stop \\
\hline Axial & $c_{\text {axial }}=1$ & 1 & 13.2 \\
Lateral & $c_{\text {radial }}=1.27$ & 0.26 & 5.24 \\
\hline
\end{tabular}

TABLE 1: Nonlinear spring characteristics (adimensional values for confidentiality).

radial direction, a single stop is enough to limit the motion of the wheel. For example, its $+\mathrm{x}$ motion is constrained by the lateral stop number 2 while the connection 1-x limits the opposite -x motion. The corresponding stiffness curves are consequently asymmetric. Dissipation is introduced in the FEM through proportional damping and local dampers to model the elastomer plots.

Sine-sweep excitation was applied locally at the bracket in different directions. The frequency band of interest spans the range from 5 to $50 \mathrm{~Hz}$ and the sweeping rate is chosen equal to four octaves per minute. This frequency range encompasses the local modes of the WEMS device and some elastic modes of the structure. More precisely, around $11 \mathrm{~Hz}$, the WEMS vibrates according to two symmetric bending modes (around $x$ and $y$ axis). Around $30 \mathrm{~Hz}$, two other symmetric modes appear combining bending (around $x$ and $y$ axis) and translation (along $x$ and $y$ axis). A mode involving the WEMS and the bracket is also present around $30 \mathrm{~Hz}$. The first lateral bending modes and the first axial mode of the structure finally appear between $30 \mathrm{and} 50 \mathrm{~Hz}$.

\section{METHODOLOGY OF IDENTIFICATION}

In this paper, we address the identification of nonlinear mechanical systems whose nonlinearities are supposed to be localized and for which there exists an underlying linear regime of vibration. The amplitude, the direction and the frequency content of the excitation determine in which regime the structure vibrates. For example, we exclude from our scope distributed or essential nonlinearities.

Such nonlinear systems are modelled through the equations

$$
\mathbf{M} \ddot{\mathbf{q}}(t)+\mathbf{C} \dot{\mathbf{q}}(t)+\mathbf{K} \mathbf{q}(t)+\sum_{j=1}^{n} W_{j} g_{j}(t)=\mathbf{p}(t)
$$

where $\mathbf{M}, \mathbf{C}$ and $\mathbf{K}$ are the mass, damping and stiffness matrices, respectively, $\mathbf{q}$ is the generalized displacement vector and $\mathbf{p}$ is the external force vector. The $n$ nonlinear forces acting on the structure are described through some weighted basis functions $g_{j}$. Our interest lies in the estimation of the weights, or the coefficients, introduced in these nonlinear expressions and denoted $W_{j}$.

We now give a practical introduction to the RFS method. Other ways to get onto this method can be found in ${ }^{[11]}{ }^{[17]}$ and ${ }^{[18]}$. First, Equation (1) is recast into

$$
\mathbf{M} \ddot{\mathbf{q}}(t)+\mathbf{f}_{n l}(t)=\mathbf{p}(t)
$$

where $\mathbf{f}_{n l}(t)$ contains all the restoring forces of the system. This offers a simple way to assess the coefficients $W_{j}$. Indeed, if we know the modelling $g_{j}(t)$ of the nonlinear forces hidden in $\mathbf{f}_{n l}(t)$, the excitation force $\mathbf{p}(t)$, an estimate of the mass matrix $\mathbf{M}$ and the kinematic signals $\mathbf{q}(t), \dot{\mathbf{q}}(t)$ and $\ddot{\mathbf{q}}(t)$, the matrix $W_{j}$ (along with $\mathbf{K}$ and $\mathbf{C}$ ) can be estimated, for instance, in a least squares sense.

For simplicity, we restrict ourselves to the underlying conservative system: neither the damping $\mathbf{C}$ of the underlying linear structure nor the localized dissipation of the elastomer plots of the WEMS will be identified. Concerning the characterizing functions $g_{j}$, the nature of the nonlinearity can guide us to its functional form. This is the case of the WEMS where a bilinear model is obvious.

The access to the excitation signal $\mathbf{p}(t)$ can appear to be trivial. However, space structures are universally tested under a base 

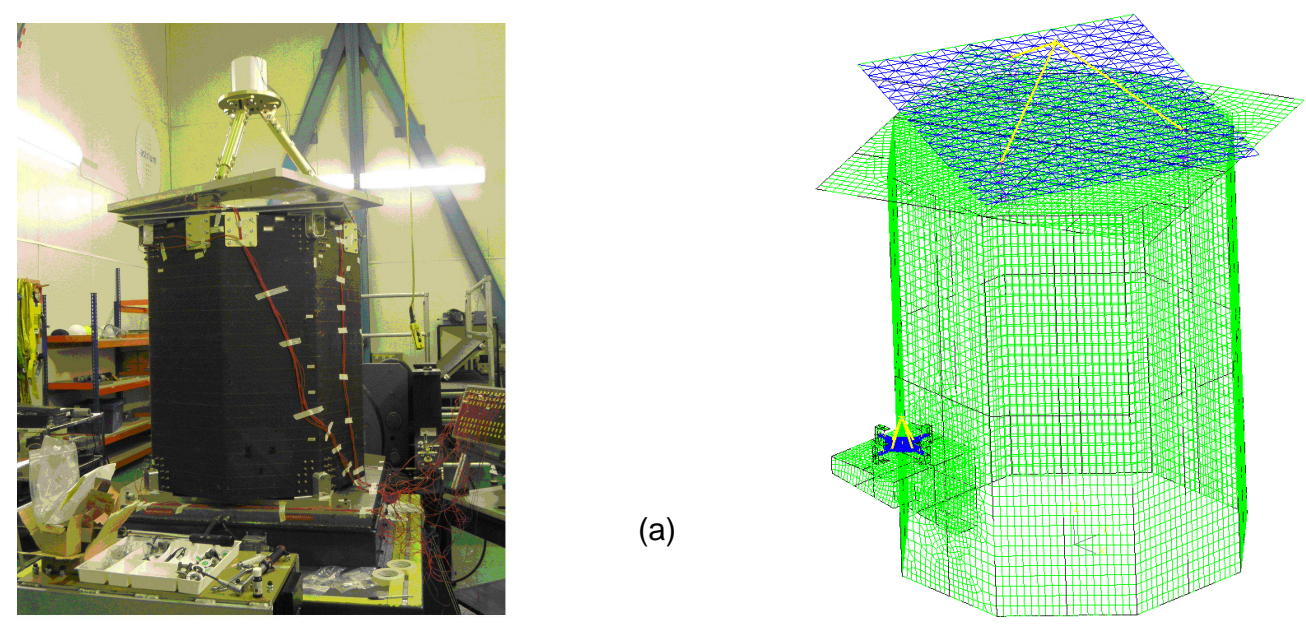

(b)
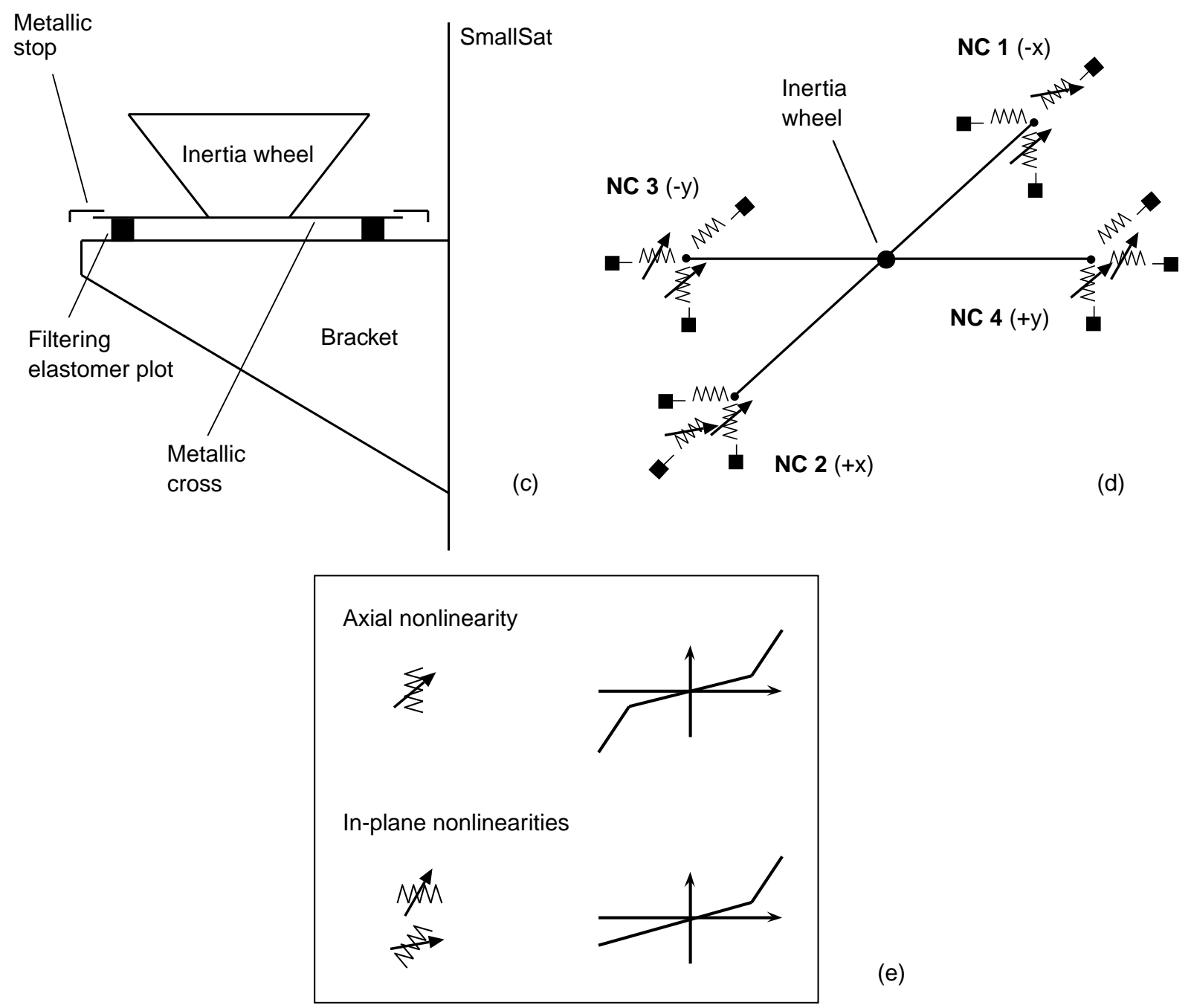

(e)

Figure 1: SmallSat structure. (a) real structure without the WEMS module; (b) finite element model; (c) WEMS module mounted on a bracket and supporting a dummy inertia wheel; (d) close-up of the WEMS mobile part (NC stands for nonlinear connection) and (e) graphical display of the nonlinear restoring forces. 
excitation for which the actual force produced by the shaker is often unknown. In our case, since the exciting force is applied locally and not directly onto the nonlinear connections, it will not complicate our identification procedure.

The practical knowledge of the mass matrix and of the kinematic signals is more questionable and will entail the whole methodology developed in this paper. Note that, in practice, only acceleration signals only are recorded. Integration and/or differentiation are then used to compute displacement $\mathbf{q}(t)$ and velocity $\dot{\mathbf{q}}(t)^{[19]}$.

\subsection{An illustrative example}

It is interesting to examine the access to these two pieces of information ( $\mathbf{M}$ and $\ddot{\mathbf{q}}(t)$ ) in the case of a simple continuous structure comprising one lumped nonlinearity. The structure of interest is here a linear clamped-free beam with a cubic nonlinear spring at its free end. This numerical set-up models the geometrical nonlinearity induced by a thin beam part positioned at the main beam free end as in ${ }^{[20]}$. Figure 2 displays the Finite Element Model (FEM) of the structure where ten 2D beam elements are considered. Each element possesses a translational (vertical) and a rotational Degree Of Freedom (DOF) denoted $\mathrm{y}_{i}$ and $\theta_{i}$, respectively. They are both numbered from 1 to 10 , the "nonlinear" DOF being $\mathrm{y}_{10}$.

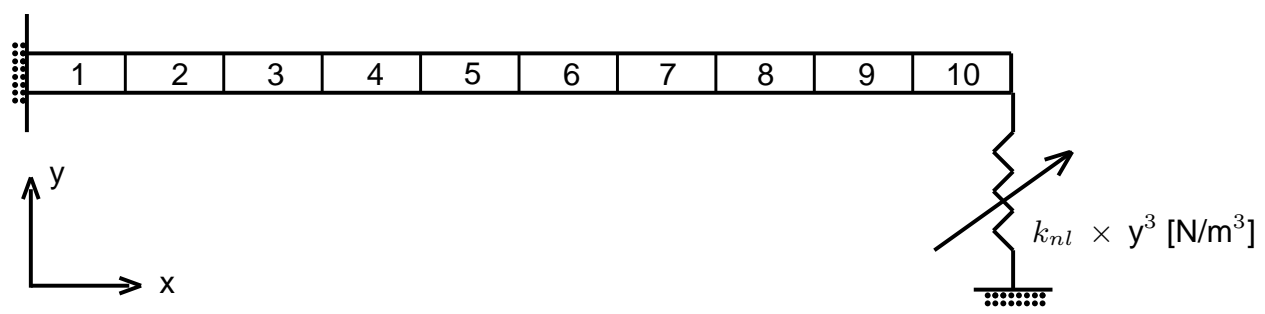

Figure 2: FEM of the nonlinear beam.

The nonlinear vibrations of that DOF are governed by the equation (assuming that there is no excitation applied at beam tip)

$$
\begin{aligned}
& \mathrm{M}_{\left[\mathrm{y}_{10}, \mathrm{y}_{9}\right]} \ddot{\mathrm{y}}_{9}(t)+\mathrm{M}_{\left[\mathrm{y}_{10}, \theta_{9}\right]} \ddot{\theta}_{9}(t)+\mathrm{M}_{\left[\mathrm{y}_{10}, \mathrm{y}_{10}\right]} \ddot{\mathrm{y}}_{10}(t)+\mathrm{M}_{\left[\mathrm{y}_{10}, \theta_{10}\right]} \ddot{\theta}_{10}(t)+\ldots \\
& \mathrm{C}_{\left[\mathrm{y}_{10}, \mathrm{y}_{9}\right]} \dot{\mathrm{y}}_{9}(t)+\mathrm{C}_{\left[\mathrm{y}_{10}, \theta_{9}\right]} \dot{\theta}_{9}(t)+\mathrm{C}_{\left[\mathrm{y}_{10}, \mathrm{y}_{10}\right]} \dot{\mathrm{y}}_{10}(t)+\mathrm{C}_{\left[\mathrm{y}_{10}, \theta_{10}\right]} \dot{\theta}_{10}(t)+\ldots \\
& \mathrm{K}_{\left[\mathrm{y}_{10}, \mathrm{y}_{9}\right]} \mathrm{y}_{9}(t)+\mathrm{K}_{\left[\mathrm{y}_{10}, \theta_{9}\right]} \theta_{9}(t)+\mathrm{K}_{\left[\mathrm{y}_{10}, \mathrm{y}_{10}\right]} \mathrm{y}_{10}(t)+\mathrm{K}_{\left[\mathrm{y}_{10}, \theta_{10}\right]} \theta_{10}(t)+k_{n l}\left(\mathrm{y}_{10}\right)^{3}=0
\end{aligned}
$$

We group the restoring forces together to finally obtain

$$
\mathrm{M}_{\left[\mathrm{y}_{10}, \mathrm{y}_{9}\right]} \ddot{\mathrm{y}}_{9}(t)+\mathrm{M}_{\left[\mathrm{y}_{10}, \theta_{9}\right]} \ddot{\theta}_{9}(t)+\mathrm{M}_{\left[\mathrm{y}_{10}, \mathrm{y}_{10}\right]} \ddot{\mathrm{y}}_{10}(t)+\mathrm{M}_{\left[\mathrm{y}_{10}, \theta_{10}\right]} \ddot{\theta}_{10}(t)+\mathbf{f}_{n l}(t)=0 .
$$

This equation shows that the computation of the restoring forces $\mathbf{f}_{n l}(t)$ requires the knowledge of the mass matrix $\mathbf{M}$ and of the accelerations measured at the translational DOF's $\mathrm{y}_{9}$ and $\mathrm{y}_{10}$ and at the rotational DOF's $\theta_{9}$ and $\theta_{10}$. Without either resorting to a FEM or complicating the experimental procedure, the access to a reliable estimate of $\mathbf{M}$ is a first serious issue. In addition, in practice, the measurement of rotational DOF's, such as $\theta_{9}$ and $\theta_{10}$, is not usually carried out.

This example immediately reveals why there exists almost no application of the RFS method to large-scale structures in the literature. Most often ${ }^{[21,22]}$, Equation (3) is truncated and adopted under the form

$$
\mathrm{M}_{\left[\mathrm{y}_{10}, \mathrm{y}_{10}\right]} \ddot{\mathrm{y}}_{10}(t)+\mathrm{C}_{\left[\mathrm{y}_{10}, \mathrm{y}_{10}\right]} \dot{\mathrm{y}}_{10}(t)+\mathrm{K}_{\left[\mathrm{y}_{10}, \mathrm{y}_{10}\right]} \mathrm{y}_{10}(t)+k_{n l}\left(\mathrm{y}_{10}\right)^{3}=0 \text {. }
$$

The scope of the method is then reduced to qualitative information, i.e. nonlinearity characterization, where it proves to be a useful tool. It is, however, no longer capable of assessing parameters. In the next subsection, we show how it is possible to 
perform RFS-based system identification of the SmallSat spacecraft under an assumption concerning the WEMS kinematics.

\subsection{Assumption of a rigid WEMS device}

One should observe that rotational DOF's, such as $\theta_{i}$ in the previous subsection, are central to describe the kinematics of a flexible body. For instance, the bending of the beam elements in Figure 2 is linked, by essence, to the rotation of their ends. On the contrary, rotations can be avoided in the description of the motion of a rigid bar element. More generaly, it is possible to completely define the kinematics of a rigid body through the measure of six translations only, without entailing rotations. It thus appears that a rigid body assumption is a way to prevent the use of unmeasurable rotational DOF's.

However, such an assumption is not applicable in the case of the clamped-free beam with cubic nonlinearity. Indeed, this latter is caused by large deflections (or deformations) of the beam and thus needs flexibility to be activated. On the other hand, several types of nonlinearity do not resort to such a flexibility in their dynamics. We can cite the geometrical nonlinearities due to large displacements which always arise in fully rigid multibody systems. This is also the case for the nonlinearities that are lumped in essence or, in other words, that are caused by localized mechanisms (e.g., friction in a bolted connection or a damper in an automotive suspension). Alternatively stated, such nonlinearities are not denatured whether the masses they connect are taken to be rigid. That is not to say that the physics of the structure itself is not modified (see next Subsection).

The WEMS case belongs to this latter class. Its bilinear behaviour in stiffness is indeed localized since it originates from the combination of lumped elements that are the elastomer plots and the stops. We consequently assume the rigidity of the inertia wheel and of its cross-shaped support. We simplify further our model by reducing the inertia wheel to a point mass whose inertia properties are allocated to the center of the cross.

To assess this rigidity assumption, we propose in Table 2 the first six elastic frequencies of the WEMS mobile part alone. Their magnitude gives sense to our approach. We will come back later on the verification of this assumption. Indeed, in the case of large vibrations, the shocks between the cross and the stops can give rise to flexible effets that have to be monitored.

\begin{tabular}{c|c} 
& Natural frequencies $(\mathrm{Hz})$ \\
\hline 1 & 3,109 \\
2 & 3,163 \\
3 & 3,175 \\
4 & 3,176 \\
5 & 6,624 \\
6 & 7,036 \\
\hline
\end{tabular}

TABLE 2: Six first elastic frequencies of the WEMS mobile part in free-free conditions.

The fourth point of the required information listing established above is fulfiled: the kinematic signals $\mathbf{q}(t), \dot{\mathbf{q}}(t)$ and $\ddot{\mathbf{q}}(t)$ are within reach. Furthermore, rigidity gives an easy access to an analytical computation of the mass matrix $\mathbf{M}$. The different elements of the WEMS as we model it were already displayed in Figure 1 (d). The rigid metallic cross and the point mass inertia wheel can thereof be seen. 


\subsection{Kinematic constraints}

The three $x-y-z$ displacements of each end of the cross (twelve in total) naturally describe the kinematic of the WEMS. Since this description requires the knowledge of six coordinates only, six of them turn out to be redundant. If the vector $q$ collects this set as

$$
\mathbf{q}^{\top}=\left(\mathrm{x}_{1} \mathrm{y}_{1} \mathrm{z}_{1} \mathrm{x}_{2} \mathrm{y}_{2} \mathrm{z}_{2} \mathrm{x}_{3} \mathrm{y}_{3} \mathrm{z}_{3} \mathrm{x}_{4} \mathrm{y}_{4} \mathrm{z}_{4}\right)
$$

it can thus be split into two subsets $\mathbf{q}_{I}$ and $\mathbf{q}_{D}$, the former containing six user-chosen independent coordinates.

As for the kinematic constraints, they express the invariance of the distance between any two points of the WEMS module. In general, this leads to a set of

$$
\frac{r !}{2 !(r-2) !}
$$

relationships. In the case of the WEMS, six distance invariants result from the last formula in agreement with the sizes of the sets $\mathbf{q}, \mathbf{q}_{\mathrm{I}}$ and $\mathbf{q}_{\mathrm{D}}$.

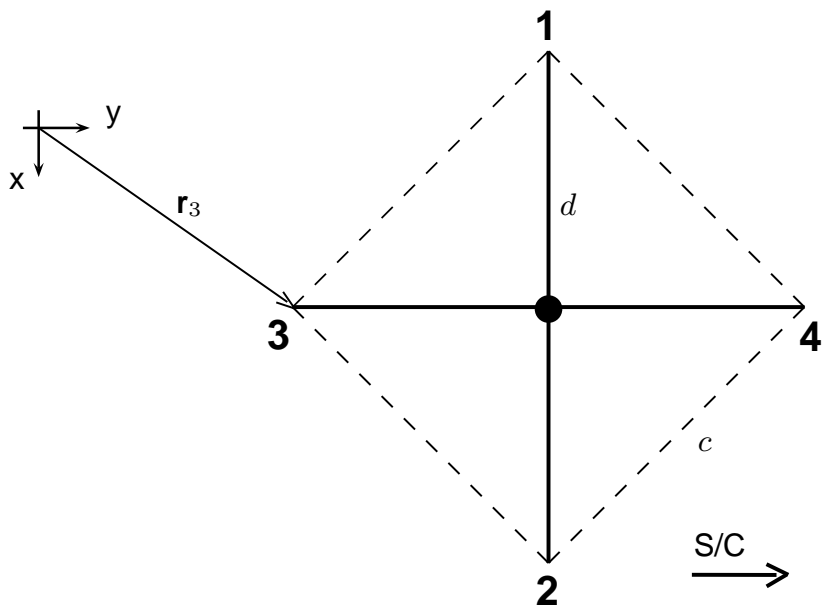

Figure 3: Top view of the square-shaped WEMS mobile part.

Considering the scheme in Figure 3, they can be formulated as

$$
\left\{\begin{array}{l}
\left\|\mathbf{r}_{1}-\mathbf{r}_{2}\right\|^{2}=d^{2} \\
\left\|\mathbf{r}_{3}-\mathbf{r}_{4}\right\|^{2}=d^{2} \\
\left\|\mathbf{r}_{2}-\mathbf{r}_{3}\right\|^{2}=c^{2} \\
\left\|\mathbf{r}_{3}-\mathbf{r}_{1}\right\|^{2}=c^{2} \\
\left\|\mathbf{r}_{1}-\mathbf{r}_{4}\right\|^{2}=c^{2} \\
\left\|\mathbf{r}_{4}-\mathbf{r}_{2}\right\|^{2}=c^{2}
\end{array}\right.
$$

where $d$ and $c$ are the length of an arm of the cross and the distance between two of its adjacent ends, respectively. These six relations indicate that the four ends of the cross are, in fact, the vertices of a square. They intrinsically express the invariability of the lengths of the two arms, their perpendicularity and their common Center of Gravity (CoG).

It is actually possible, but herein skipped, to demonstrate that the set of constraints (8) can be transformed into the more intuitive relationships of length, orthogonality and coincidence

$$
\left\{\begin{array}{ccc}
\left\|\mathbf{r}_{1}-\mathbf{r}_{2}\right\|^{2} & = & d^{2} \\
\left\|\mathbf{r}_{3}-\mathbf{r}_{4}\right\|^{2} & = & d^{2} \\
\left(\mathbf{r}_{1}-\mathbf{r}_{2}\right) \cdot\left(\mathbf{r}_{3}-\mathbf{r}_{4}\right) & = & 0 \\
\left(\mathbf{r}_{1}+\mathbf{r}_{2}\right) / 2 & = & \left(\mathbf{r}_{3}+\mathbf{r}_{4}\right) / 2 .
\end{array}\right.
$$


Already at this stage, we want to underline the mathematical complexity of these relations. Their impossibility to explicitely define $\mathbf{q}_{\mathrm{D}}$, no matter its definition, will play a major role further in this paper.

In the previous Subsection, we gave in a first argument in favour of the rigid body assumption (in terms of elastic frequencies). We can herein make use of the geometrical conditions of rigidity to control on-line the quality of our assumption. This is central as our approach would loose its suitability in case of flexibility effects. Indeed, significant terms in Equations 1 would then be erroneously neglected.

To that purpose, we formulate the aforementioned geometrical constraints in terms of relative errors and seek potential deviations from rigidity during the increase of the excitation frequency:

$$
\left\{\begin{array}{ccc}
\left\|\mathbf{r}_{1}-\mathbf{r}_{2}\right\|^{2} / d^{2}-1 & = & 0 \\
\left\|\mathbf{r}_{3}-\mathbf{r}_{4}\right\|^{2} / d^{2}-1 & = & 0 \\
\cos ^{-1}\left(\frac{\left(\mathbf{r}_{1}-\mathbf{r}_{2}\right) \cdot\left(\mathbf{r}_{3}+\mathbf{r}_{4}\right)}{\left\|\mathbf{r}_{1}-\mathbf{r}_{2}\right\|^{2}\left\|\mathbf{r}_{3}+\mathbf{r}_{4}\right\|^{2}}\right) \frac{2}{\pi}-1 & = & 0 \\
\left(\mathbf{r}_{1}+\mathbf{r}_{2}\right) /\left(\mathbf{r}_{3}+\mathbf{r}_{4}\right)-1 & = & 0 .
\end{array}\right.
$$

In Figure 4, we propose a first example of this verification means ( $\mathrm{z}$ excitation on the bracket at $300 \mathrm{~N}$ ). At this excitation level, the system is nonlinear. As intuitively expected, the perpendicularity is almost exactly verified. The $4-z$ stop is actually reached and this is visible in the deviations of the second and sixth constraints (explained by impacts on the stops). The influence of the resonances of the structure are also clearly detectable on these six plots.

We can inspect a second set of constraints under $\mathrm{x}$ excitation at $300 \mathrm{~N}$ (for which the system is now linear) in Figure 5 . Their verification is improved mainly on the sixth constraint. This highlights the role of the impacts in the relevancy of the rigid body assumption.

In conclusion, we see that our geometrical verification approach provides a qualitative measure of the confidence in our identification strategy, and therefore in the subsequently estimated coefficients.

\subsection{Explicit formulation of the WEMS dynamics}

We already explained in Subsection 3.1 that a rigorous and thorough writing of Newton's law of motion is crucial to the RFS method. It is worth pointing out that an unconstrained form of these equations is also obviously sought. Whereas the writing of such a form is direct for classical vibrating structures, the situation gets more complicated in the presence of kinematic constraints. This problem is addressed in the present Subsection.

First, we explicitely introduce the elastic restoring forces in the system through the trilinear form (for which we assume the asymmetry of the WEMS)

$$
\mathrm{f}_{n l}\left(q_{i}\right)=\left\{\begin{array}{ccc}
\left(k_{i}^{0}+k_{i}^{1}\right) q_{i}^{r}+k_{i}^{1} d_{i}^{1} & \text { if } & q_{i}^{r}<-d_{i}^{1} \\
k_{i}^{0} q_{i}^{r} & \text { if } & -d_{i}^{1} \leq q_{i}^{r} \leq d_{i}^{2} \\
\left(k_{i}^{0}+k_{i}^{2}\right) q_{i}^{r}-k_{i}^{2} d_{i}^{2} & \text { if } & q_{i}^{r}>d_{i}^{2}
\end{array}\right.
$$

where

- $q_{i}$ is the $i^{\text {th }}$ component of $\mathbf{q}$,

- $k_{i}^{0}, k_{i}^{1}$ and $k_{i}^{2}$ are the stiffnesses of the elastomer and of the stops, respectively, 

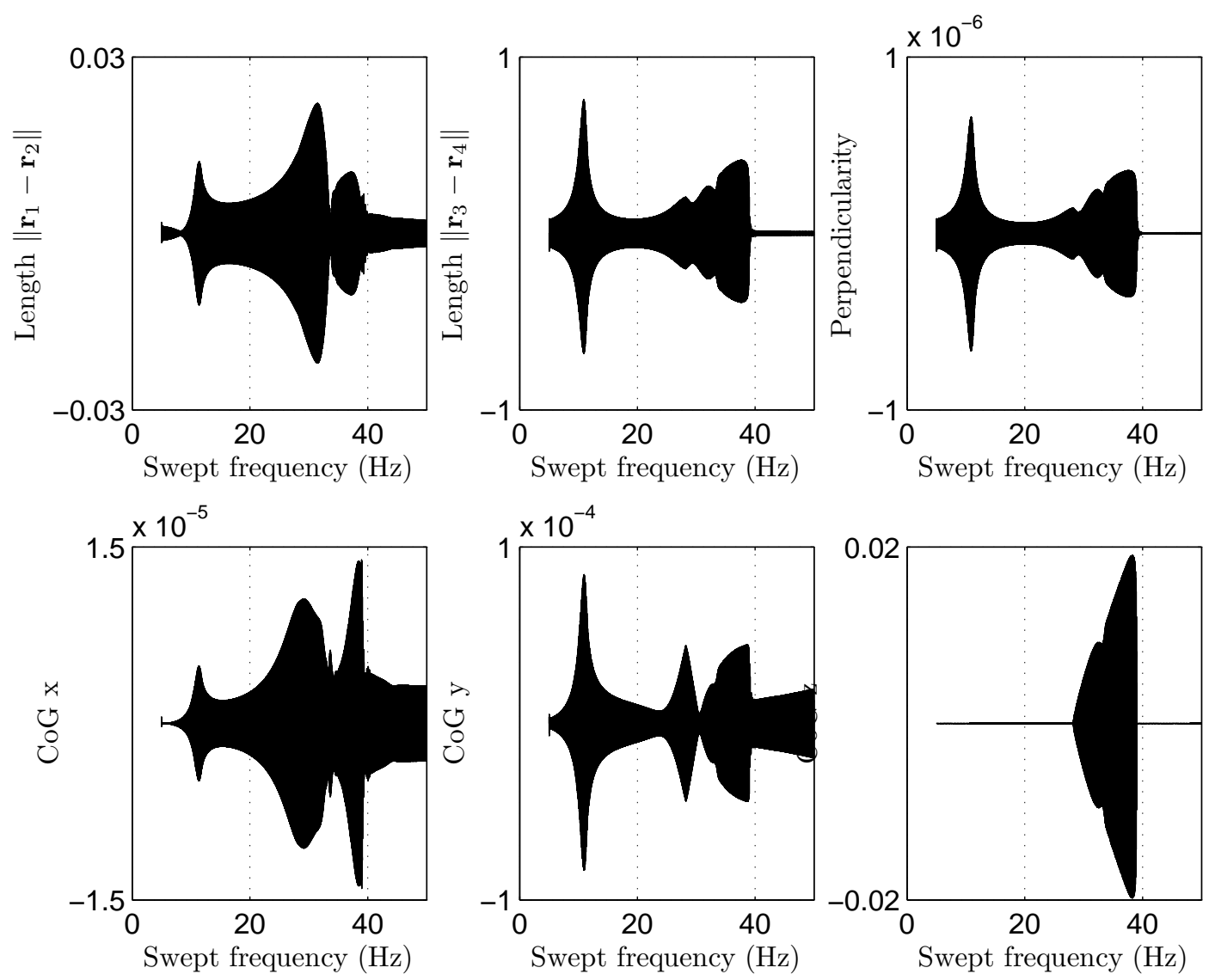

Figure 4: On-line verification (in percents) of the geometrical conditions of rigidity at $300 \mathrm{~N} \mathrm{(z} \mathrm{excitation).}$

- $d_{i}^{1}$ and $d_{i}^{2}$ are the associated clearances.

Figure 6 displays this stiffness curve. Note that we use the notation $q_{i}^{r}$ rather than $q_{i}$ to remind that the force in the springs is linked to the relative motion of their ends. The relative displacement $q_{i}^{r}$ thus designates the difference between two opposite displacements of the fixed and mobile parts of the WEMS. We also draw attention to the connections noted 1-y, 2-y, 3-x and $4-x$ that are linear and for which we simply write

$$
\mathrm{f}_{n l}\left(q_{i}\right)=k_{i}^{0} q_{i}^{r}
$$

In general, the expression of the elastic forces in the WEMS can be shortened following

$$
\mathrm{f}_{n l}\left(q_{i}\right)=k_{i} q_{i}^{r}+k_{i}^{*}
$$

where $k_{i}$ and $k_{i}^{*}$ are piecewise constant.

The requirement for reaching unconstrained equations of motion is the possibility to free them from $\mathbf{q}^{\mathrm{D}}$ by substitution. In other words, the requirement is an explicit knowledge of the relation

$$
\mathbf{q}^{\mathrm{D}}=\Phi\left(\mathbf{q}^{\mathrm{I}}\right)
$$

Such a relation is out of reach because of the complexity of the set of constraints (9). This can be clarified by considering the 

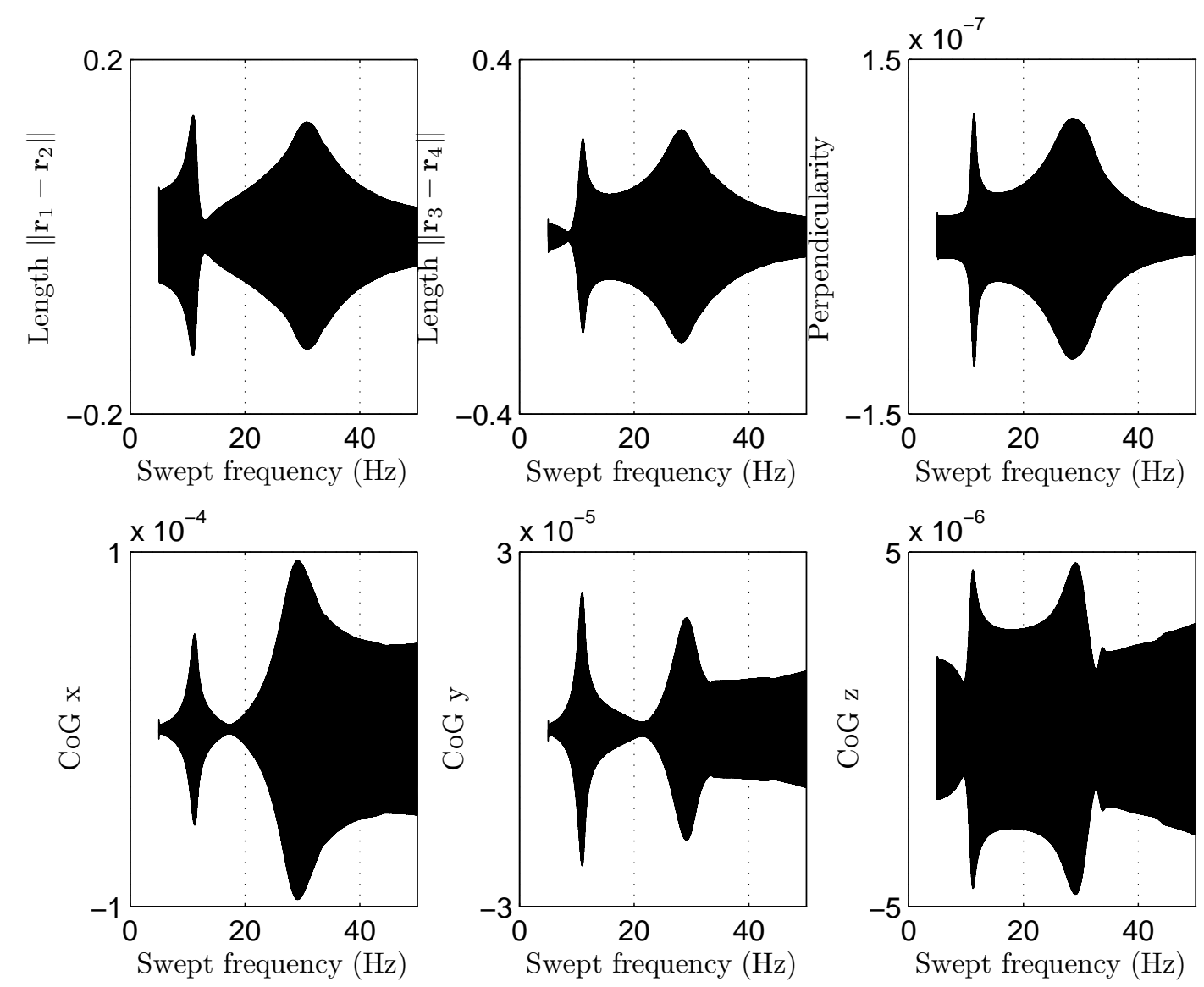

Figure 5: On-line verification (in percents) of the geometrical conditions of rigidity at $300 \mathrm{~N}$ (x excitation).

writing of the potential energy in the system. This energy, stored in the twelve linear and nonlinear stiffnesses, has the form

$$
V=V(\mathbf{q})=\sum_{i=1}^{12} \frac{1}{2} k_{i}\left(q_{i}^{r}\right)^{2}+k_{i}^{*} q_{i}^{r} .
$$

After elimination of $\mathbf{q}^{\mathrm{D}}$, we should reach its unconstrained expression, depending on $\mathbf{q}^{\mathrm{I}}$ only,

$$
V^{*}=V^{*}\left(\mathbf{q}^{\mathrm{I}}\right) \text {. }
$$

More particularly, we are interested in the elastic forces which require its gradient, in the Lagrange's formalism,

$$
\frac{\partial V^{*}}{\partial \mathbf{q}^{\mathrm{I}}}=\frac{\partial V}{\partial \mathbf{q}} \frac{\partial \mathbf{q}}{\partial \mathbf{q}^{\mathrm{I}}} .
$$

We can detail this latter as

$$
\frac{\partial V^{*}}{\partial q_{j}^{\mathrm{I}}}=\sum_{i: q_{i} \in \mathbf{q}^{\mathrm{I}}} \frac{\partial V}{\partial q_{i}} \delta_{i j}+\sum_{i: q_{i} \in \mathbf{q}^{\mathrm{D}}} \frac{\partial V}{\partial q_{i}} \frac{\partial q_{i}}{\partial q_{j}^{\mathrm{I}}}
$$

where it becomes obvious that the last derivate cannot be computed. Indeed, it needs the explicit relationship (14) while we only possess its implicit definition (9) of the form

$$
\Psi\left(\mathbf{q}^{\mathrm{I}}, \mathbf{q}^{\mathrm{D}}\right)=0
$$

We are consequently compelled to choose a new describing set of independant coordinates and we naturally turn to the CoG of the WEMS mobile part. We formulate the new definition

$$
\mathbf{q}^{\mathrm{I}}=\left(\mathrm{x}_{\mathrm{CoG}} \mathrm{y}_{\mathrm{CoG}} \mathrm{z}_{\mathrm{CoG}} \alpha \beta \gamma\right)^{\top}
$$




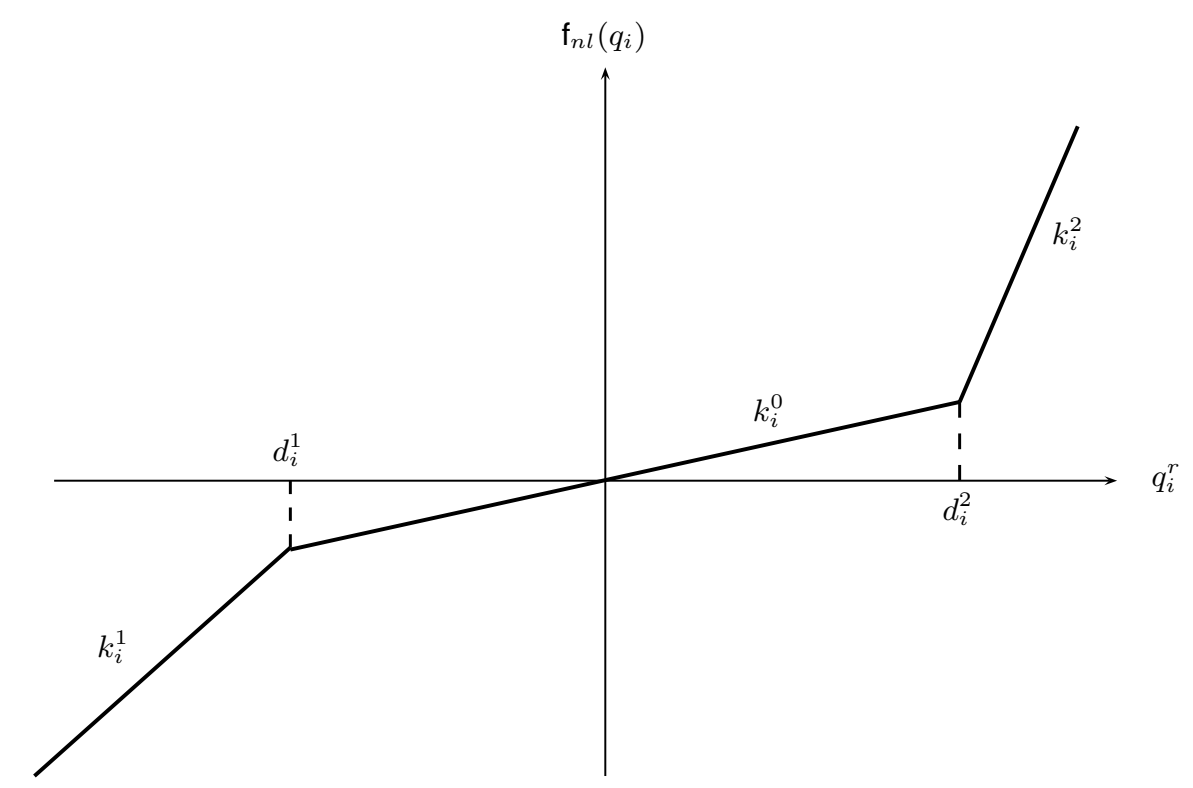

Figure 6: Bilinear (or here trilinear since asymmetry is supposed) force to be identified.

where $\mathrm{x}_{\mathrm{CoG}}, \mathrm{y}_{\mathrm{CoG}}$ and $\mathrm{z}_{\mathrm{CoG}}$ measure the translation of the $\mathrm{CoG}$ in the $\mathrm{x}-\mathrm{y}-\mathrm{z}$ frame and $\alpha, \beta$ and $\gamma$ parametrize its rotation. We also redefine $\mathbf{q}^{\mathrm{D}}$ as

$$
\mathbf{q}^{\mathrm{D}}=\left(\begin{array}{lllllllllllllll}
\mathrm{x}_{1} & \mathrm{y}_{1} & \mathrm{z}_{1} & \mathrm{x}_{2} & \mathrm{y}_{2} & \mathrm{z}_{2} & \mathrm{x}_{3} & \mathrm{y}_{3} & \mathrm{z}_{3} & \mathrm{x}_{4} & \mathrm{y}_{4} & \mathrm{z}_{4}
\end{array}\right)^{\top}
$$

and $\mathbf{q}$ as $\mathbf{q}^{\mathrm{I}} \cap \mathbf{q}^{\mathrm{D}}$.

The immediate advantage of this choice is the possibility to write the transformation $\mathbf{q}^{\mathrm{D}}=\Phi\left(\mathbf{q}^{\mathrm{I}}\right)$ as

$$
\left(\begin{array}{l}
\mathrm{x}_{i} \\
\mathrm{y}_{i} \\
\mathrm{z}_{i}
\end{array}\right)=\left(\begin{array}{l}
\mathrm{x}_{\mathrm{CoG}} \\
\mathrm{y}_{\mathrm{CoG}} \\
\mathrm{z}_{\mathrm{CoG}}
\end{array}\right)+\mathbf{R}(\alpha, \beta, \gamma)\left(\begin{array}{c}
\mathrm{X}_{i} \\
\mathrm{Y}_{i} \\
\mathrm{Z}_{i}
\end{array}\right), \quad i=1, \ldots, 4
$$

where $\left(\mathrm{X}_{i} \mathrm{Y}_{i} \mathrm{Z}_{i}\right)^{\top}$ is the initial (undeformed) position of one end of the cross and $\mathbf{R}$ is a rotation matrix.

In the parametrization of the WEMS rotation, we opt for Bryant angles because of their intuitive interpretation. The roll and pitch angles directly quantify rotations around the $x$ and $y$ arms of the cross. As for the yaw angle, it corresponds to a linear torsion of the WEMS around $z$ axis.

One can go back to the computation of the elastic forces and write, since the potentiel energy $V$ (see Equation (15)) is independant of the CoG coordinates,

$$
\frac{\partial V}{\partial q_{j}^{\mathrm{I}}}=\sum_{i: q_{i} \in \mathbf{q}^{\mathrm{D}}} \frac{\partial V}{\partial q_{i}} \frac{\partial q_{i}}{\partial q_{j}^{\mathrm{I}}}
$$

It thus appears that the restoring force $\partial V / \partial q_{j}^{\mathrm{I}}$ can be computed from the product between the gradient $\partial V / \partial q_{i}$ of $V$ and the $j^{\text {th }}$ column of the Jacobian $J$ associated with the set of Equations (22).

In addition, the kinetic energy in the system takes the simple form

$$
T=\frac{1}{2}\left(m \dot{\mathrm{x}}_{C o G}^{2}+m \dot{\mathrm{y}}_{C o G}^{2}+m \dot{\mathrm{z}}_{C o G}^{2}+I_{x} \dot{\alpha}^{2}+I_{y} \dot{\beta}^{2}+I_{z} \dot{\gamma}^{2}\right)
$$


and leads to the diagonal mass matrix

$$
\mathbf{M}=\left(\begin{array}{cccccc}
m & & & & & \\
& m & & & 0 & \\
& & m & & & \\
& & & I_{x} & & \\
& 0 & & & I_{y} & \\
& & & & & I_{z}
\end{array}\right) .
$$

Under the rigid body assumption, the following unconstrained equation describes the motion of the WEMS rigourously and is suited to a RFS-based parameter estimation:

$$
\mathbf{M}_{j} \ddot{q}_{j}^{\mathrm{I}}+\vec{\nabla} V \cdot J_{j}=0
$$

where the coefficients to be assessed are hidden in $\vec{\nabla} V$ and where $\mathbf{M}_{j}$ and $J_{j}$ designate the $j^{\text {th }}$ diagonal term of $\mathbf{M}$ and column of $J$, respectively. It can be shown that the first three scalar Equations (26) give the access to the estimation of the twelve stiffnesses of the system since they write

$$
\left\{\begin{array}{l}
m \ddot{\mathbf{x}}_{C o G}+\mathrm{f}_{n l}\left(\mathbf{x}_{1}\right)+\mathrm{f}_{n l}\left(\mathbf{x}_{2}\right)+\mathrm{f}_{n l}\left(\mathbf{x}_{3}\right)+\mathrm{f}_{n l}\left(\mathbf{x}_{4}\right)=0 \\
m \ddot{\mathbf{y}}_{C o G}+\mathrm{f}_{n l}\left(\mathbf{y}_{1}\right)+\mathrm{f}_{n l}\left(\mathbf{y}_{2}\right)+\mathrm{f}_{n l}\left(\mathbf{y}_{3}\right)+\mathrm{f}_{n l}\left(\mathbf{y}_{4}\right)=0 \\
m \ddot{\mathbf{z}}_{C o G}+\mathrm{f}_{n l}\left(\mathbf{z}_{1}\right)+\mathrm{f}_{n l}\left(\mathbf{z}_{2}\right)+\mathrm{f}_{n l}\left(\mathbf{z}_{3}\right)+\mathrm{f}_{n l}\left(\mathbf{z}_{4}\right)=0 .
\end{array}\right.
$$

We will consequently restrict our results to the use of these three equations. Rigidity rises a last issue, discussed in the following subsection, and linked to the rank deficiency of the matrix $\vec{\nabla} V \cdot J$.

\subsection{Identification of a rigid body: an ill-conditioned problem}

Let us consider the identification of the WEMS stiffnesses in the $x$ direction and in linear regime. The first of the Equations (27) then becomes

$$
m \ddot{\mathrm{x}}_{C o G}+k_{\mathrm{x}_{1}}^{0} \mathbf{x}_{1}^{r}+k_{\mathrm{x}_{2}}^{0} \mathrm{x}_{2}^{r}+k_{\mathrm{x}_{3}}^{0} \mathrm{x}_{3}^{r}+k_{\mathrm{x}_{4}}^{0} \mathbf{x}_{4}^{r}=0 .
$$

For the purpose of the identification, this equation is written as a least squares problem:

$$
\underbrace{\left(\mathbf{x}_{1}^{r} \mathbf{x}_{2}^{r} \mathbf{x}_{3}^{r} \mathbf{x}_{4}^{r}\right)}_{\mathbf{A}} \underbrace{\left(\begin{array}{llll}
k_{\mathrm{x}_{1}}^{0} & k_{\mathrm{x}_{2}}^{0} & k_{\mathrm{x}_{3}}^{0} & k_{\mathrm{x}_{4}}^{0}
\end{array}\right)^{\top}}_{\mathbf{X}}=\underbrace{-m \ddot{\mathbf{x}}_{C o G}}_{\mathbf{b}} .
$$

In practice, matrix A turns out to be badly conditioned preventing an accurate estimation of the coefficients $k_{\mathrm{x}_{i}}^{0}$. More precisely, this matrix appears to be not of full rank. The explanation is twofold. On the one hand, and according the fourth and last Equation of constraint (9), there exists a linear relationship between the displacements of the WEMS mobile part considered direction per direction. On the other hand, since the WEMS fixed part (actually the bracket) is almost at rest in our frequency band of interest $(5-50 \mathrm{~Hz})$, this linear dependance is not altered when moving to relative displacements as in matrix $\mathbf{A}$.

As noted in the literature ${ }^{[23]}$, without perturbations and rounding errors, the solution to the rank-deficient system of equations $\mathbf{A} \mathbf{x}=\mathbf{b}$ is straightforward. Indeed, if we introduce the Singular Value Decomposition (SVD) of $\mathbf{A}$

$$
\mathbf{A}=\sum_{i=1}^{n} u_{i} \sigma_{i} v_{i}^{\top}
$$

this solution writes

$$
\mathbf{x}^{\text {ideal }}=\sum_{i=1}^{\operatorname{rank}(\mathbf{A})} \frac{u_{i}^{\top} \mathbf{b}}{\sigma_{i}} v_{i}
$$

However, practically speaking, $\mathbf{A}$ is never exactly rank deficient since it has one or several small but non-zero singular values ${ }^{[23]}$. It is said to be numerically rank deficient and causes the aforementioned least squares problem to be ill-conditioned, i.e. its solution is dominated by the errors. A simple regularization strategy consists in truncating the singular value spectrum of $\mathbf{A}$ and 
thus replacing its smallest elements with exact zeros. In other words, $\mathbf{A}$ is seen as a noisy representation of the mathematically rank deficient matrix $\mathbf{A}_{k}$ defined as

$$
\mathbf{A}_{k}=\sum_{i=1}^{k<n} u_{i} \sigma_{i} v_{i}^{\top} .
$$

The stiffness coefficients of the WEMS are then computed in a numerically stable way through

$$
\mathbf{x}=\mathbf{A}_{k}^{\dagger} \mathbf{b}=\sum_{i=1}^{k} \frac{u_{i}^{\top} \mathbf{b}}{\sigma_{i}} v_{i}
$$

where $\dagger$ denotes the inverse of a rectangular matrix in a least squares sense. This approach is known as the Truncated Singular Value Decomposition (TSVD). Its difficulty lies in the choice of $k$, i.e. the number of sufficiently large singular values, that is to say in the definition of what a small singular value is.

\section{IDENTIFICATION RESULTS}

We first consider an axial ( $\mathrm{z}$ ) excitation applied to the WEMS bracket at $200 \mathrm{~N}$. At this excitation level, no mechanical stop is reached and the dynamics thus remains linear. Our interest lies in the estimation of the axial stiffnesses since the relative displacements in the lateral directions are negligible. Figure 7 presents our results, summarized in Table 3 . The estimation is of high quality as proved by a simple visual inspection of the force-displacement curves.

\begin{tabular}{c|c|c|c|c|c}
\hline Identification case & & $1-\mathrm{z}$ & $2-\mathrm{z}$ & $3-\mathrm{z}$ & $4-\mathrm{z}$ \\
\hline $200 \mathrm{~N}$ & Linear est. & 1.00 & 1.06 & 1.03 & 1.02 \\
(linear estim.) & & $0.26 \%$ & $5.50 \%$ & $2.55 \%$ & $2.12 \%$ \\
\hline $300 \mathrm{~N}$ & Nonlinear est. & - & - & - & 12.93 \\
(only nonlinear estim.) & & & & & $2.08 \%$ \\
\hline $300 \mathrm{~N}$ & Linear est. & 1.66 & 0.84 & 0.82 & 0.80 \\
(full estim.) & & $66.29 \%$ & $16.41 \%$ & $17.79 \%$ & $20.03 \%$ \\
& Nonlinear est. & - & - & - & 12.90 \\
& & & & & $2.32 \%$ \\
\hline $400 \mathrm{~N}$ & Nonlinear est. & - & - & 12.26 & 12.29 \\
(only nonlinear estim.) & & & & $7.14 \%$ & $6.92 \%$ \\
\hline $500 \mathrm{~N}$ & Nonlinear est. & - & - & 13.92 & 13.10 \\
(only nonlinear estim.) & & & & $5.41 \%$ & $0.78 \%$ \\
\hline
\end{tabular}

TABLE 3: Summary of the identification results in the axial direction. Each estimate is given with a relative deviation from its exact value.

Figure 8 shows a qualitative application of the RFS method. As explained in Section 3.1, this simplified approach constitutes a powerful characterization tool. However, it cannot be used for parameter estimation purposes since it is based on truncated equations of motion, as obvious in this figure.

As a last result in the linear case, we prove the relevancy of a TSVD regularization in Table 4. The four singular values of the matrix $\mathbf{A}$ to be inverted in our least squares resolution present a large gap between the second and the third ones. It reveals a numerical rank equal to 2 . The consequent truncation of this singular value spectrum led us to the results in Figure 7.

Prior to addressing a nonlinear case, we anticipate a discussion about displacement coupling tackled later in this paper. The confidence factor ${ }^{[17]}$ shown in Figure 4 shows that there is no contribution of the lateral motion in the fitting of the axial restoring forces.

We now move to a $300 \mathrm{~N}$ excitation level. The axial nonlinear connection numbered $4-\mathrm{z}$ is activated when sweeping the modes of vibration around $30 \mathrm{~Hz}$. This activation is clear on the corresponding time series (Figure 10) where a jump phenomenon is 

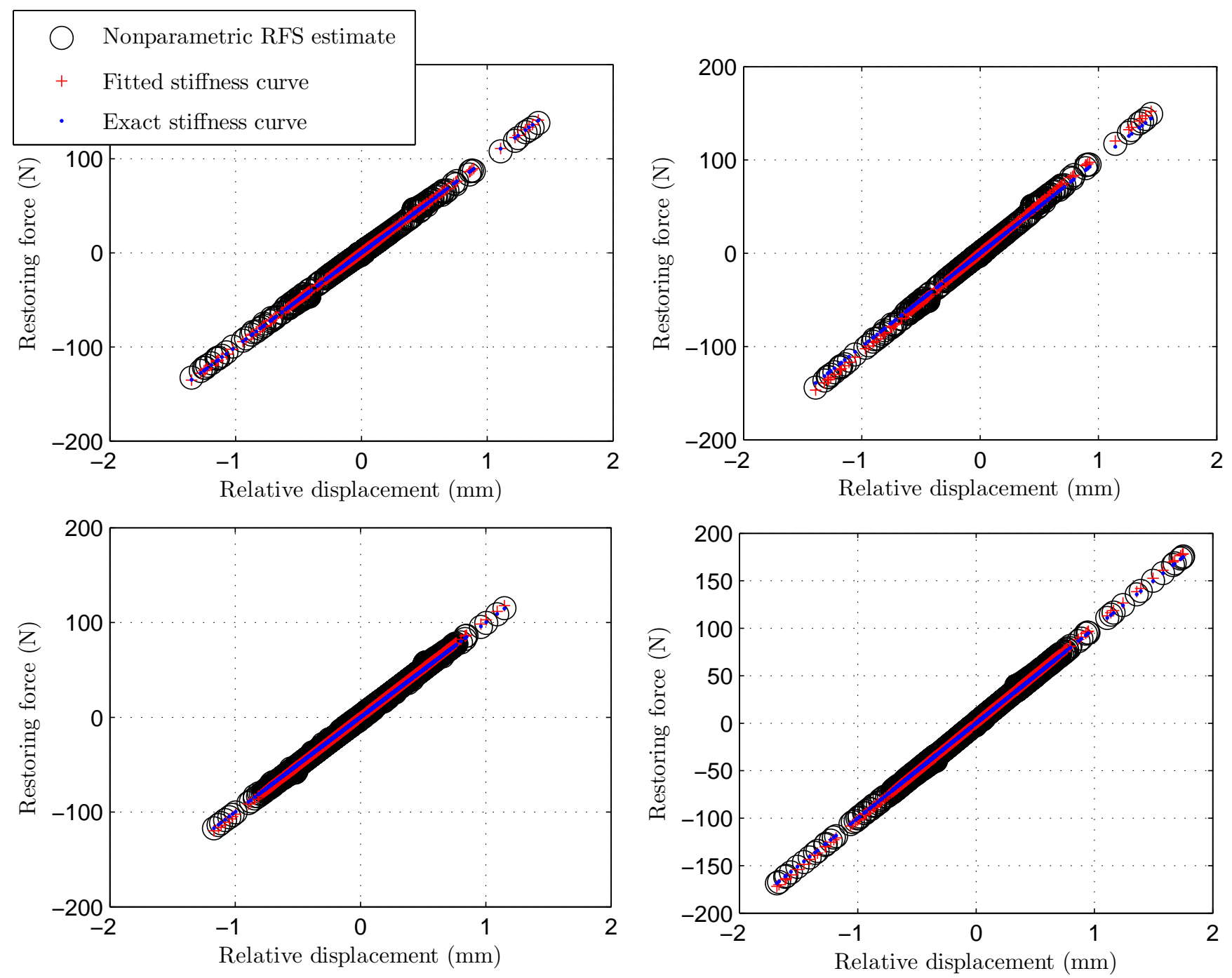

Figure 7: Parametric estimation of the stiffnesses 1-z (top left), 2-z (top right), 3-z (bottom left) and 4-z (bottom right).

\begin{tabular}{c|c}
$200 \mathrm{~N}$ axial case & Singular value $(\%)$ \\
\hline 1 & 55.6 \\
2 & 43.3 \\
3 & 0.6 \\
4 & 0.5 \\
\hline
\end{tabular}

TABLE 4: The third and fourth singular values should be equal to zero. They differ because of rounding errors.

highlighted.

We chose to assess the 4-z nonlinear stiffness using the four linear estimates obtained in the previous step (Figure 7). This approach turns out to be the most accurate. Our result is depicted in Figure 11 and also listed in Table 3. Here again, we note an excellent agreement with the exact result (relative error of $2 \%$ ). We can however try to simultaneously fit both the four linear 


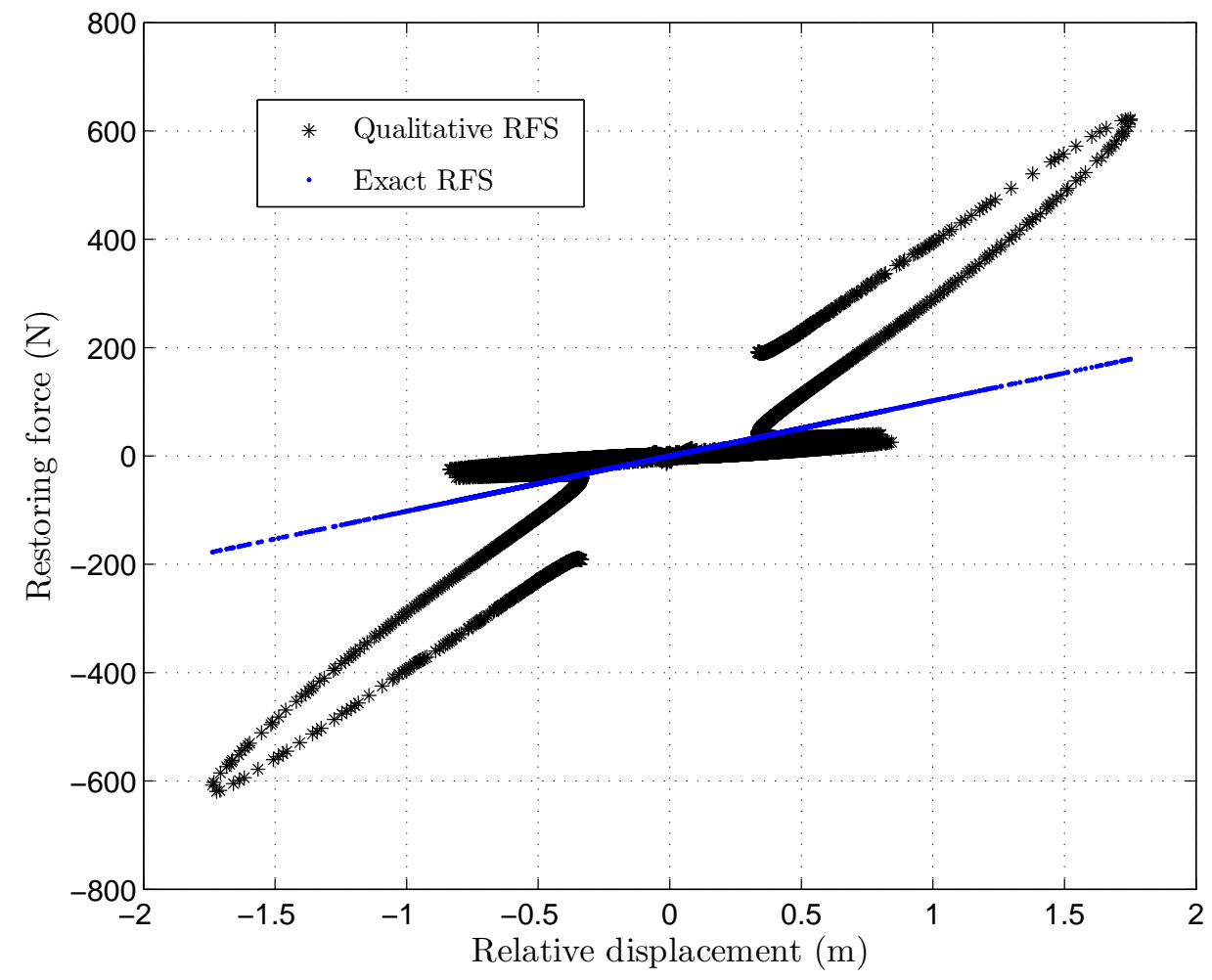

Figure 8: Qualitative application of the RFS method to the 4-z connection. The different WEMS modes in the frequency band of excitation appear with various and inexact slopes.

and the nonlinear forces (next case in Table 3). The nonlinear estimate remains of high quality whereas the linear parameters get worse. This gives sense to our decoupled estimation.

We finally address $400 \mathrm{~N}$ and $500 \mathrm{~N}$ excitations and focus on the first modes of the WEMS around $11 \mathrm{~Hz}$. Indeed, considering their shapes, the modes of vibration around $30 \mathrm{~Hz}$ cannot lead to the activation of several nonlinearities. The reach of the 4-z nonlinear regime actually prevents other stops from being impacted. This explains our interest in the first modes which are activated at higher energy but involve two nonlinear restoring forces. Here again, we exploit our aforementioned linear estimates in order to focus on a nonlinear estimation problem. The estimates take place in Table 3 and a graphical display at $500 \mathrm{~N}$ is given in Figure 12. As one may expect, the higher the level of activation of the nonlinearities, the more accurate their estimation.

The estimation of the lateral stiffnesses (under a lateral excitation) turns out to be much more challenging and will clearly underline the limitations of our RFS-based identification strategy. Their explanation lies in the existence of kinematic couplings.

To detail this issue, we now excite the structure along an oblique direction with respect to the $\mathrm{x}$ and $\mathrm{y}$ reference axis (at the linear $200 \mathrm{~N}$ regime) and try to estimate the y-stiffness coefficients. The result provided in Table 5 is of unsatisfactory quality. This quality can be enhanced by considering the existence of kinematic couplings. To that purpose, we include in our fitting basis all the available $x, y$ and $z$ restoring forces as potential candidates. The estimation is depicted in Figure 13 and summarized in Table 5. Its quality appears to be acceptable and the analysis of the stiffness curves confirms that judgement. The need for an extended fitting panel is obvious in Figure 14. In particular, the 3-z and 4-z contributions are more relevant than the y stiffnesses themselves. Finally, we stress that a much improved quality would require additional fitting terms (mainly modelling dissipation) as demonstrated by the remaining disturbances on the force-displacement curves (Figure 13).

Another proof of interdependence between the directions of motion of the WEMS is given in Figure 15 (x excitation). This restor- 


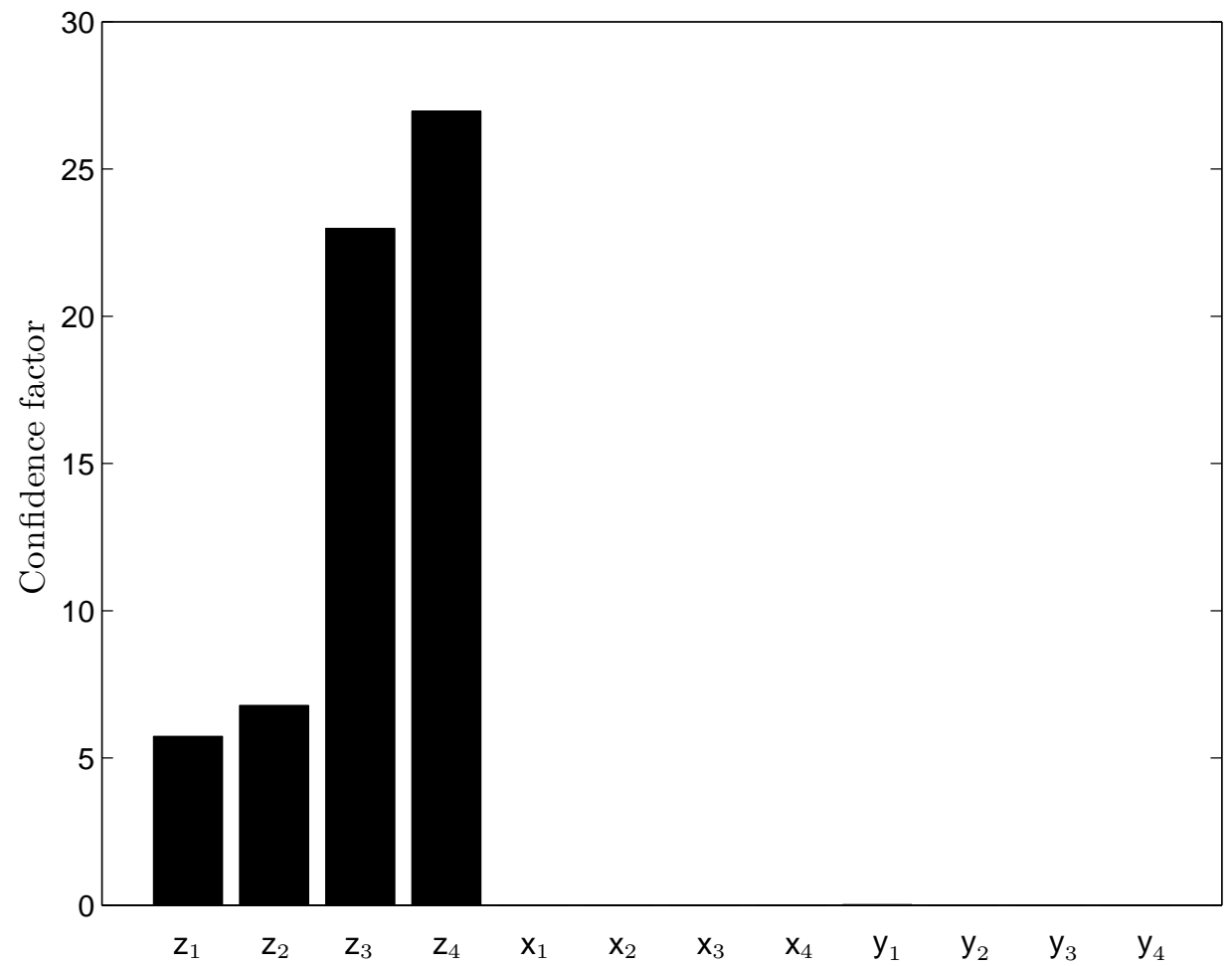

Figure 9: The recourse to lateral contributions appears to be useless in axial identification.
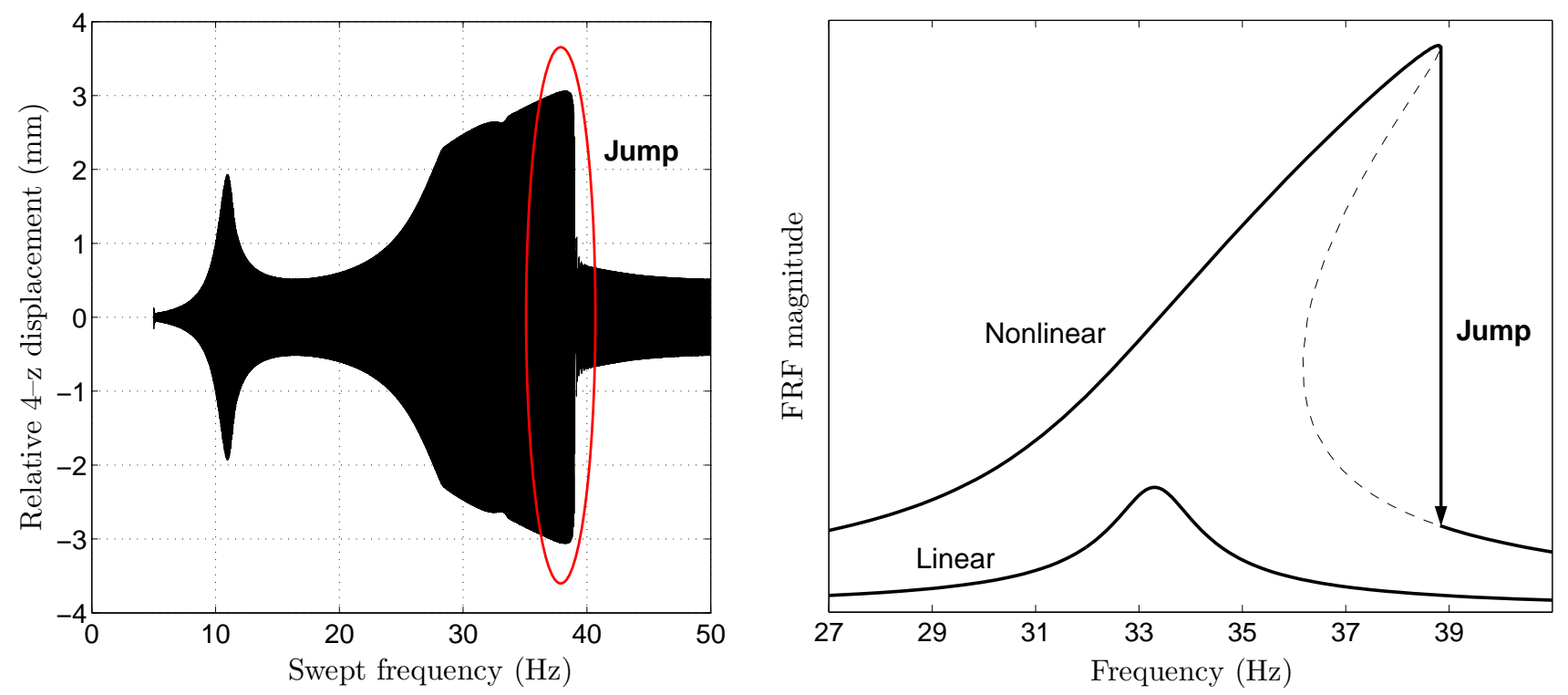

Figure 10: Jump phenomenon caused by the distortion of the corresponding FRF. 


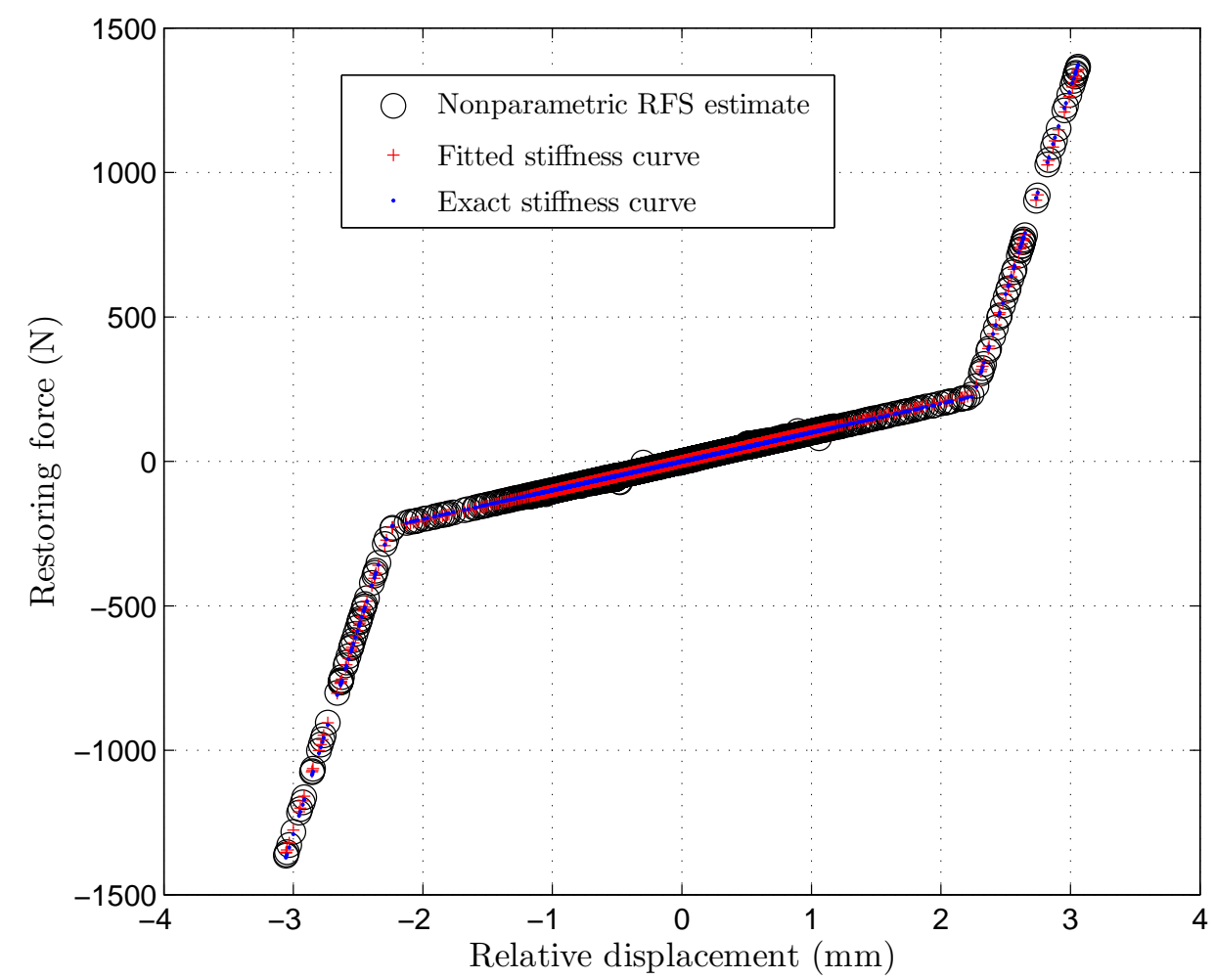

Figure 11: Excellent estimation of the 4-z nonlinear force.

\begin{tabular}{c|c|c|c|c|c}
\hline Identification case & & $1-\mathrm{y}$ & $2-\mathrm{y}$ & $3-\mathrm{y}$ & $4-\mathrm{y}$ \\
\hline $200 \mathrm{~N}$ & Linear est. & -0.73 & 0.96 & 0.12 & 0.12 \\
(linear estim.) & & $379.42 \%$ & $268.95 \%$ & $55.18 \%$ & $55.37 \%$ \\
\hline $200 \mathrm{~N}$ & Linear est. & 0.32 & 0.24 & 0.28 & 0.28 \\
(extended basis) & & $22.83 \%$ & $6.75 \%$ & $7.00 \%$ & $8.00 \%$ \\
\hline
\end{tabular}

TABLE 5: Summary of the identification results in the y lateral direction. Each estimate is given with a relative deviation from its exact value.

ing force curve reveals a trilinear behaviour. Such a form is actually caused by the simultaneous reach of an axial and a lateral stop. Their appearance on a single force-displacement curve is indeed an additional proof of coupling.

An experimental campaign was also performed on the SmallSat spacecraft, and the results are currently analysed. A preliminary result in Figure 16 clearly highlights the piecewise linear nature of the WEMS device.

\section{CONCLUSIONS}

This paper aimed at applying the Restoring Force Surface method to a real-life space structure: the SmallSat spacecraft. The elimination of kinematic constraints and the regularization of ill-conditioned inverse problems are the two main contributions of the study. To date, the identification results are promising. A particular attention should be devoted to damping contributions in a further work. A possible approach would be a non-physical modelling through Fourier series. In addition, the SmallSat 

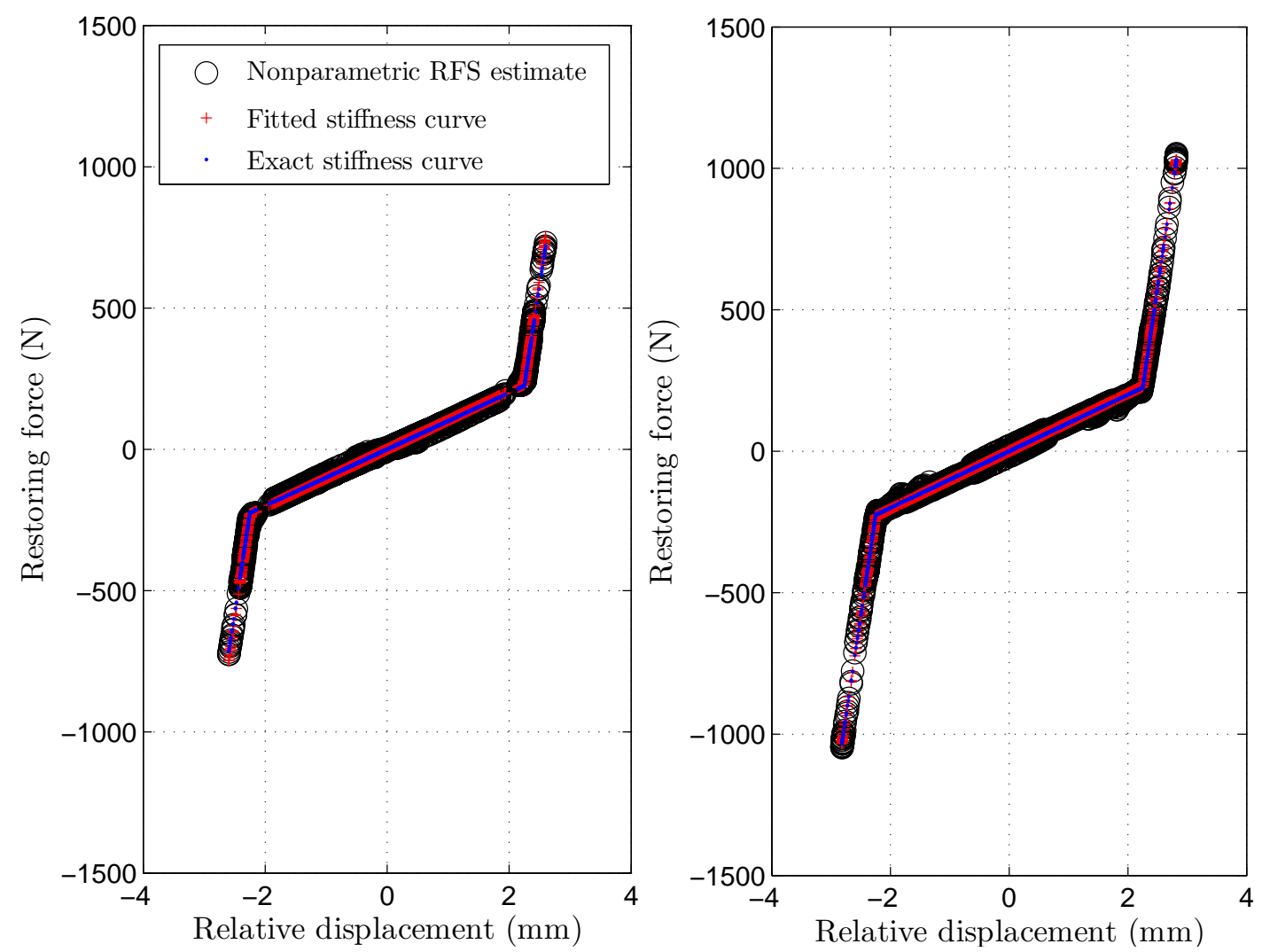

Figure 12: Simultaneous identification of the 3-z (left) and 4-z (right) restoring forces.

identification from experimental data is also in progress.

\section{ACKNOWLEDGMENTS}

This paper has been prepared in the framework of the ESA Technology Research Programme study "Advancement of Mechanical Verification Methods for Non-linear Spacecraft Structures (NOLISS)" (ESA contract No.21359/08/NL/SFe).

The author J.P. Noël would like to acknowledge the Belgian National Fund for Scientific Research (FRIA fellowship) for its financial support.

The authors finally thank Astrium SAS for sharing information about the SmallSat spacecraft. 

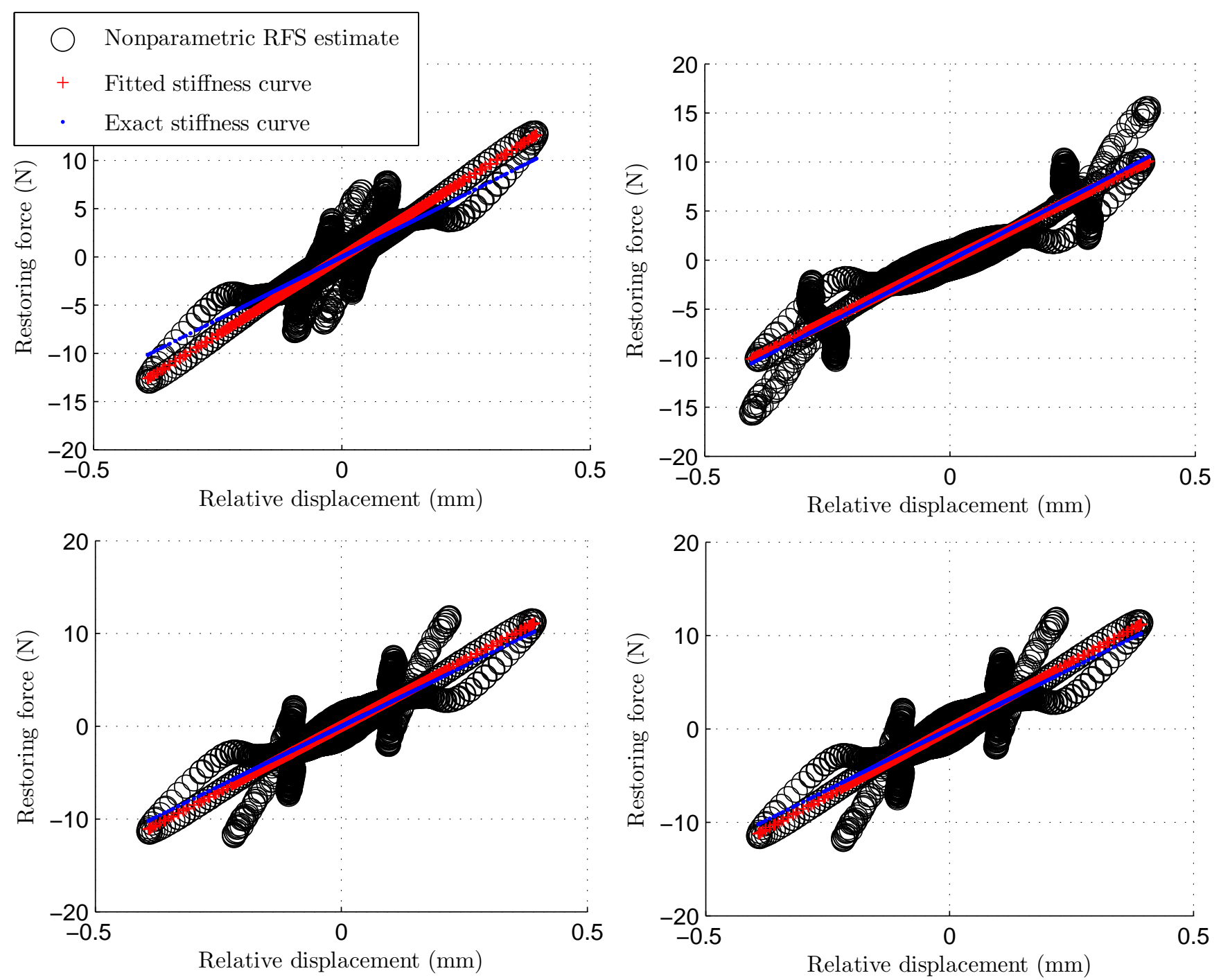

Figure 13: Parametric estimation of the stiffnesses 1-y (top left), 2-y (top right), 3-y (bottom left) and 4-y (bottom right). 


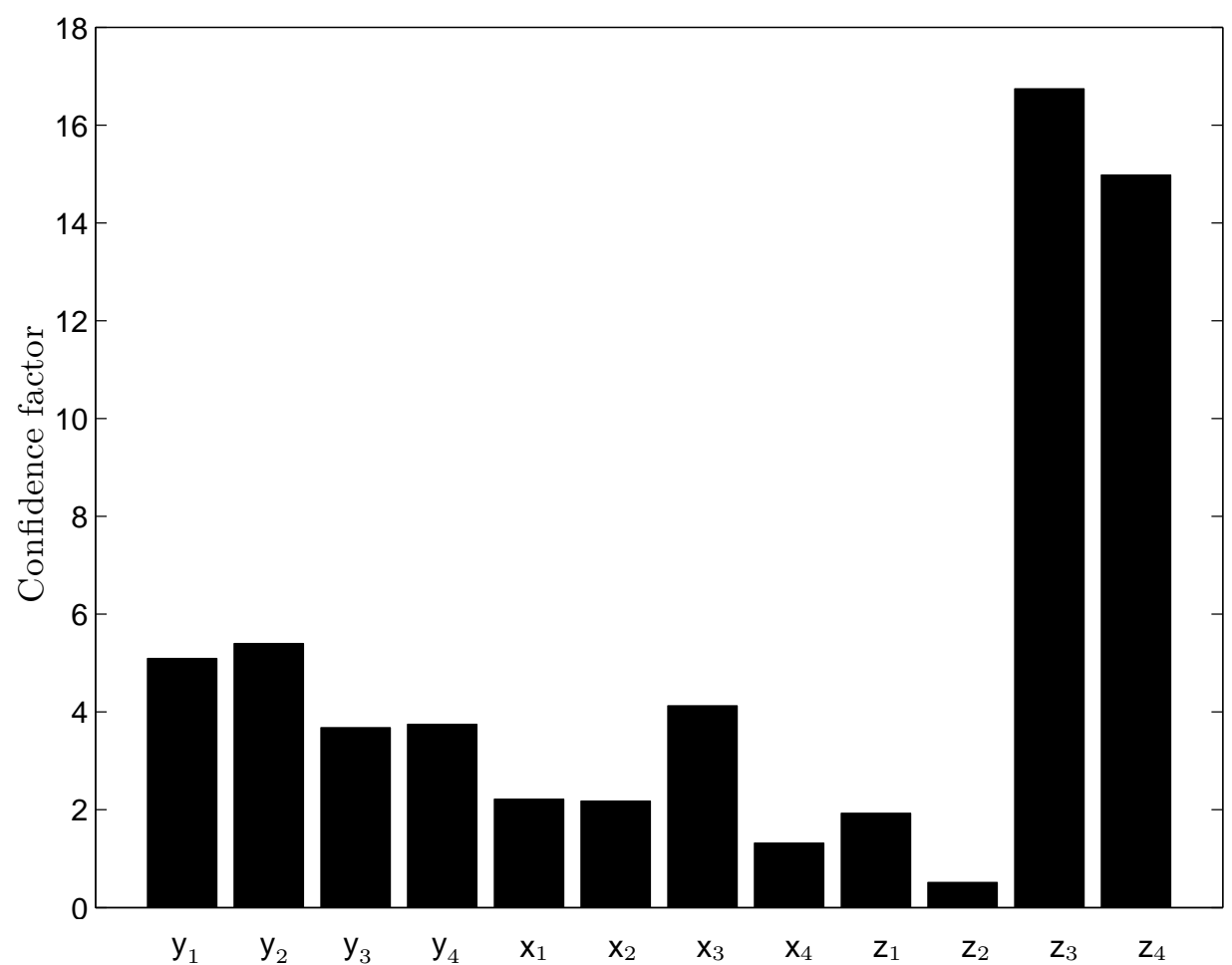

Figure 14: The whole fitting basis is relevant in the case of the $y$ identification.

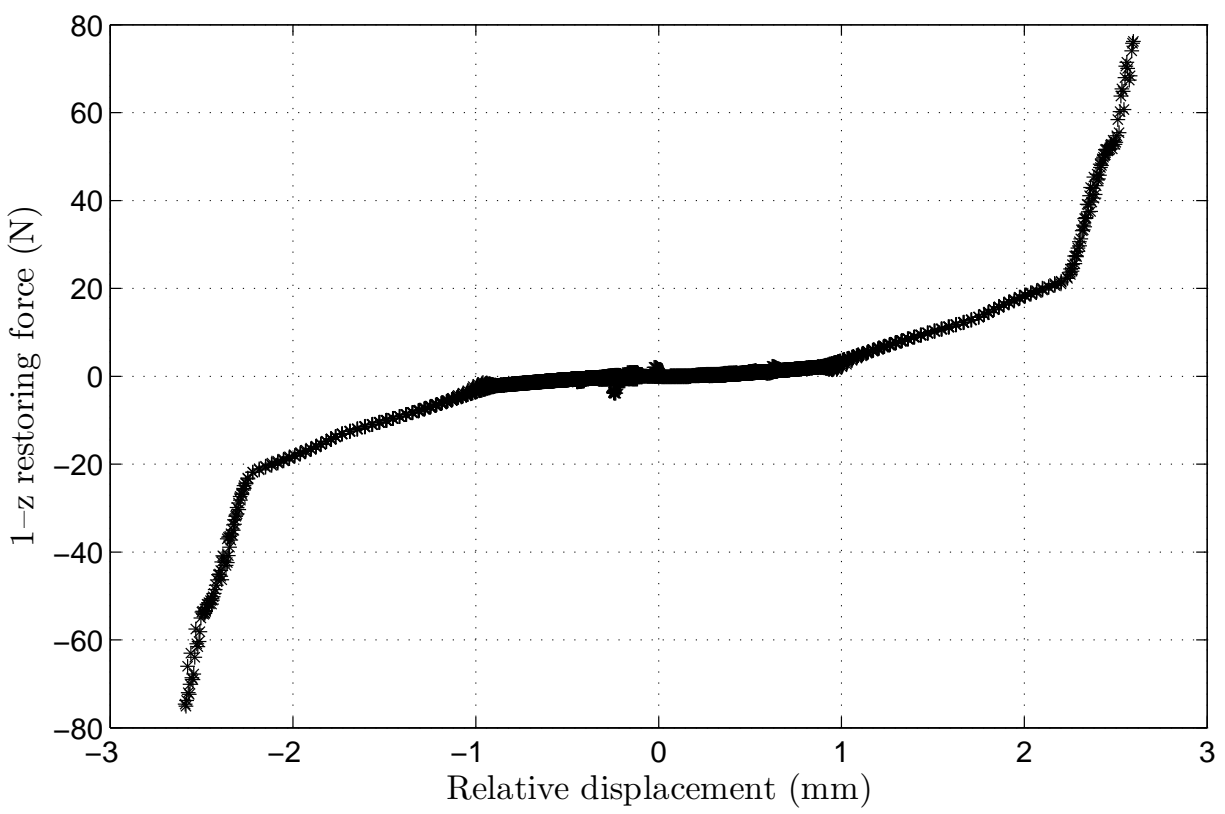

Figure 15: The reach of two perpendicular stops induces a coupled trilinear behaviour. 


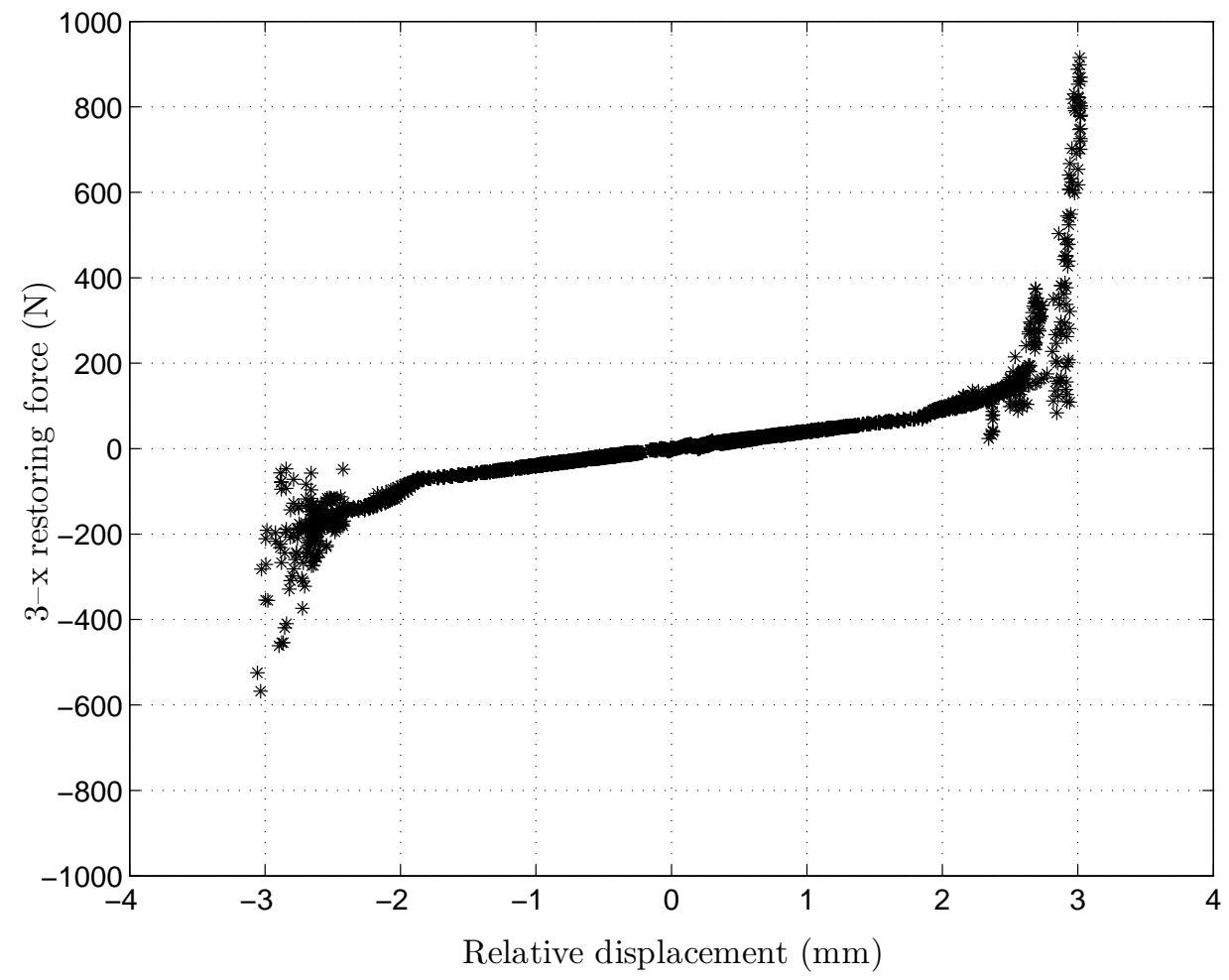

Figure 16: A first qualitative restoring force computed on experimental measurements. 


\section{REFERENCES}

[1] Kerschen, G., Worden, K., Vakakis, A. F. and Golinval, J. C., Past, present and future of nonlinear system identification in structural dynamics, Mechanical Systems and Signal Processing, 2006.

[2] Leontaritis, I. J. and Billings, S. A., Input-output parametric models for nonlinear systems, part I: deterministic nonlinear systems, International Journal of Control, 1985.

[3] Leontaritis, I. J. and Billings, S. A., Input-output parametric models for nonlinear systems, part II: stochastic nonlinear systems, International Journal of Control, 1985.

[4] Richards, C. M. and Singh, R., Identification of multi-degree-of-freedom non-linear systems under random excitations by the reverse-path spectral method, Journal of Sound and Vibration, 1998.

[5] Adams, D. E. and Allemang, R. J., A frequency domain method for estimating the parameters of a non-linear structural dynamic model through feedback, Mechanical Systems and Signal Processing, 2000.

[6] Ruzzene, M., Fasana, A., Garibaldi, L. and Piombo, B., Natural frequencies and dampings identification using wavelet transform: application to real data, Mechanical Systems and Signal Processing, 1997.

[7] Masri, S. F. and Caughey, T. K., A nonparametric identification technique for nonlinear dynamic problems, Journal of Applied Mechanics, 1979.

[8] Yang, Y. and Ibrahim, S. R., A nonparametric identification technique for a variety of discrete nonlinear vibrating systems, ASME Journal of Vibration, Acoustics, Stress and Reliability in Design, 1985.

[9] Duym, S. and Schoukens, J., Design of excitation signals for the restoring force surface method, Mechanical Systems and Signal Processing, 1995.

[10] Crawley, E. F. and O'Donnell, K. J., Identification of nonlinear system parameters in joints using the force-state mapping technique, AIAA Paper, 1986.

[11] Worden, K. and Tomlinson, G. R., Nonlinearity in Structural Dynamics: Detection, Identification and Modelling, Taylor and Francis, 2001.

[12] Al-Hadid, M. A. and Wright, J. R., Application of the Force-State Mapping Approach to the Identification of Nonlinear Systems, Mechanical Systems and Signal Processing, 1992.

[13] Worden, K. and Tomlinson, G. R., Parametric and Nonparametric Identification of Automotive Shock Absorbers, International Modal Analysis Conference (IMAC) X, 1992.

[14] Worden, K. et al., Nonlinear system identification of automotive dampers: A time and frequency-domain analysis, Mechanical Systems and Signal Processing, 2008.

[15] Russell, A. G., Thick skin, faceted, CFRP, monocoque tube structure for smallsats, European Conference on Spacecraft Structures, Materials and Mechanical Testing, 2000.

[16] Camarasa, P. and Kiryenko, S., Shock attenuation system for spacecraft and adaptor (SASSA), European Conference on Spacecraft Structures, Materials and Mechanical Testing, 2009.

[17] Kerschen, G., Golinval, J. C. and Worden, K., Theoretical and Experimental Identification of a Non-linear Beam, Journal of Sound and Vibration, 2001.

[18] Duym, S. W. R., Nonparametric Identification of Nonlinear Mechanical Systems, Ph.D. thesis, Vrije Universiteit Brussel, 1998.

[19] Worden, K., Data Processing and Experiment Design for the Restoring Force Surface Method, Part I: Integration and Differentiation of Measured Time Data, Mechanical Systems and Signal Processing, 1990.

[20] Kerschen, G., Lenaerts, V. and Golinval, J. C., Identification of a continuous structure wth a geometrical non-linearity. Part I: Conditioned Reverse Path, Journal of Sound and Vibration, 2003.

[21] Kerschen, G., Soula, L., Vergniaud, J. B. and Newerla, A., Assessment of Nonlinear System Identification Methods using the SmallSat Spacecraft Structure, International Modal Analysis Conference (IMAC) XXIX, 2011. 
[22] Peeters, M., Kerschen, G., Golinval, J. C., Stephan, C. and Lubrina, P., Nonlinear Normal Modes of a Full-Scale Aircraft, International Modal Analysis Conference (IMAC) XXIX, 2011.

[23] Hansen, P. C., Rank-Deficient and Discrete III-Posed Problems, Ph.D. thesis, Technical University of Denmark, 1996. 